\title{
A system of nonlinear fractional BVPs with $\phi$-Laplacian operators and nonlocal conditions
}

\author{
Bahia Temar ${ }^{1} \odot$ orcid.org/0000-0001-7493-5325 \\ Ouiza Saifi ${ }^{2}$ \\ Smaïl Djebaliºrcid.org/0000-0002-2318-1989
}

Ecole Normale Supérieure Cheikh Mohamed El-Bachir El-Ibrahimi, Laboratoire "Théorie du Point Fixe et Applications", Kouba, Algiers, Algeria.

1ⓣemarbahia@gmail.com; ${ }^{\circledR}$ saifi_rayen@hotmail.com; ${ }^{3}$ djebali@hotmail.com

\section{Abstract:}

This work investigates the existence of multiple positive solutions for a system of two nonlinear higher-order fractional differential equations with $\phi$-Laplacian operators and nonlocal conditions. A degenerate nonlinearity which obeys some general growth conditions is considered. The singularities are dealt with by approximating the fixed point operator. New existence results are presented by using the fixed point index theory. Examples of applications illustrate the theoretical results.

Keywords: Fixed point index; Positive solution; Fractional differential equation; Nonlocal boundary condition; $\phi$-Laplacian.

MSC (2020): 34A08, 34B10, 34B18.

\section{Cite this article as (IEEE citation style):}

B. Temar, 0. Saifi, and S. Djebali, "A system of nonlinear fractional BVPs with $\phi$-Laplacian operators and nonlocal conditions", Proyecciones (Antofagasta, On line), vol. 40, no. 2, pp. 447-479, 2021, doi: 10.22199/issn.0717-6279-2021-020027

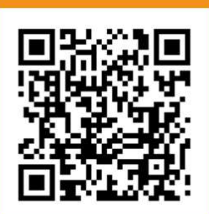

Article copyright: (C) 2021 Bahia Temar, Ouiza Saifi and Smail Djebali. This is an open access article distributed under the terms of the Creative Commons License, which permits unrestricted use and distribution provided the original author and source are credited. 


\section{Introduction}

Fractional differential equations are much more adequate and more realistic than integer-order differential equations to model several problems in science and engineering. These equations appear in many problems arising from physics, engineering, and dynamical systems but also from fluid flows, electrical networks, dynamics of earthquakes, ... because they seem to agree with experimental studies. This has motivated several researchers to use the fractional modelling in some recent research works in applied mathematics. In addition, several homogeneous or non-homogeneous boundary conditions (BCs for brevity) have been adopted in problem modeling, ranging from linear to nonlinear boundary conditions; actually they reveal information on the behaviour of the unknowns (temperature, displacement, velocity, ...) on boundaries of strings, rods, and plates (e.g., heat transfer across isolated surfaces according to Newton's law of cooling). By nonlocal BCs, it is meant here multi-point $\mathrm{BCs}$, integral BCs, or more general differential and/or algebraic BCs.

In $[8,10,14]$, we can find a detailed discussion of some fractional equations and systems of equations arising in physical phenomena. As for fractional calculus and main properties of fractional derivative operators, we refer the reader to $[7,11,9]$.

From a mathematical point of view, a differential operator (with a fractional or an integer order) may be extended to $p$-Laplacian order operators. Notice that the latter is only linear for $p=2$. The case of the $\phi$-Laplacian is again a more general extension of the $p$-Laplacian. This is our main motivation to consider in this work such general fractional operator. More precisely, we consider the existence of positive solutions for the following system of two nonlinear higher-order fractional differential equations with $\phi$-Laplacian operators and nonlocal BCs:

$$
\left\{\begin{array}{l}
-D_{0^{+}}^{\alpha_{1}}\left(\phi_{1}\left(D_{0^{+}}^{\beta_{1}} x(t)\right)=a_{1}(t) f(t, x(t), y(t)), t \in(0,1)\right. \\
-D_{0^{+}}^{\alpha_{2}}\left(\phi_{2}\left(D_{0^{+}}^{\beta_{2}} y(t)\right)=a_{2}(t) g(t, x(t), y(t)), t \in(0,1)\right. \\
x(0)=x(1)=x^{\prime}(0)=x^{\prime}(1)=0, D_{0^{+}}^{\beta_{1}} x(0)=0, D_{0^{+}}^{\beta_{1}} x(1)=b_{1} D_{0^{+}}^{\beta_{1}} x\left(\mu_{1}\right), \\
y(0)=y(1)=y^{\prime}(0)=y^{\prime}(1)=0, D_{0^{+}}^{\beta_{2}} y(0)=0, D_{0^{+}}^{\beta_{2}} y(1)=b_{2} D_{0^{+}}^{\beta_{2}} y\left(\mu_{2}\right),
\end{array}\right.
$$

where $\alpha_{i} \in(1,2], \beta_{i} \in(3,4]$. Throughout this paper, $D_{0^{+}}^{\alpha}$ will denote the standard Riemann-Liouville derivative of order $\alpha$.

For $i=1,2$, the coefficient functions $b_{i} \in\left(0, \phi_{i}^{-1}\left(\mu_{i}^{1-\alpha_{i}}\right)\right)$ and $a_{i} \in$ $C([0,1])$ while $\mu_{i} \in(0,1)$. The nonlinearities $f=f(t, x, y), g=g(t, x, y)$ : 
$[0,1] \times \mathbf{R}_{*}^{+2} \longrightarrow \mathbf{R}^{+}$are continuous and degenerate at $x=0$ and $y=0$. For $i=1,2$, the mappings $\phi_{i}: \mathbf{R} \longrightarrow \mathbf{R}$ are continuous homeomorphisms that are increasing and multiplicative, i.e., $\left(\phi_{i}(x y)=\phi_{i}(x) \phi_{i}(y), \forall x, y>\right.$ $0)$, with $\phi_{i}(0)=0$. Here and hereafter, we will use the notations $\mathbf{R}^{+}=$ $[0,+\infty)$ and $\mathbf{R}_{*}^{+}=(0,+\infty) . C([0,1])$ and $L([0,1])$ will refer to the space of continuous functions and to the class of Lebesgue integrable functions, respectively. Indeed, we are here interested in classical solutions which is sufficient for our purpose for the nonlinearities do not depend on first derivatives.

The positivity of solutions is physically interpreted by the fact that some parameters and unknowns such as temperature, density, displacement, ... are positive data. The higher-order derivative operator (which encompasses second-order operators) used in each equation is motivated by the investigation here of BVPs (boundary value problems) for a coupling system instead of IVPs (initial value problems). To avoid heavy fixed point fractional operator, we have considered homogeneaous BCs for solutions and derivatives and nonlocal BCs for higher fractional derivatives. Recall further that, in physics, the nonlinear functions $f, g$ may stand for source terms and have behavior which range from polynomial to exponential growth. The nonlinearities considered in this work encompass some growth conditions adopted in recent papers. Next, we are going to describe briefly some of them.

In the particular case of the $p$-Laplacian, namely $\varphi_{p}(s)=|s|^{p-2} s(p>$ 1 ), the authors of [6] discussed the following system of nonlinear fractional differential equations subject to nonlocal BCs:

$$
\left\{\begin{array}{l}
-D_{0^{+}}^{\alpha_{1}}\left(\varphi_{p_{1}}\left(D_{0^{+}}^{\beta_{1}} u(t)\right)=\lambda f(t, u(t), v(t)), 0<t<1\right. \\
-D_{0^{+}}^{\alpha_{2}}\left(\varphi_{p_{2}}\left(D_{0^{+}}^{\beta_{2}} v(t)\right)=\mu g(t, u(t), v(t)), 0<t<1\right. \\
u(0)=u(1)=u^{\prime}(0)=u^{\prime}(1)=0, D_{0^{+}}^{\beta_{1}} u(0)=0, D_{0^{+}}^{\beta_{1}} u(1)=b_{1} D_{0^{+}}^{\beta_{1}} v\left(\eta_{1}\right), \\
v(0)=v(1)=v^{\prime}(0)=v^{\prime}(1)=0, D_{0^{+}}^{\beta_{2}} v(0)=0, D_{0^{+}}^{\beta_{2}} v(1)=b_{2} D_{0^{+}}^{\beta_{2}} v\left(\eta_{2}\right),
\end{array}\right.
$$

where $\alpha_{i} \in(1,2], \beta_{i} \in(3,4], \eta_{i} \in(0,1)$, and $b_{i} \in\left(0, \eta_{i}^{\frac{1-\alpha_{i}}{p_{i}-1}}\right), \quad i=1,2$. The nonlinearities $f, g \in C\left([0,1] \times[0,+\infty)^{2},[0,+\infty)\right)$. According to the positive parameters $\lambda, \mu$, the existence of positive solutions is obtained by making use of the Guo-Krasnosel'skii fixed point theorem. The authors have considered classes of superlinear and sublinear functions $f, g$.

In [12], the authors considered the following fractional three-point BVP: 


$$
\left\{\begin{array}{l}
-D_{0^{+}}^{\alpha_{1}}\left(\varphi_{p}\left(D_{0^{+}}^{\beta_{1}} u(t)\right)=f(t, u(t)), 0<t<1,\right. \\
u(0)=u(1)=u^{\prime}(0)=u^{\prime}(1)=0, D_{0^{+}}^{\beta_{1}} u(0)=0, D_{0^{+}}^{\beta_{1}} u(1)=b D_{0^{+}}^{\beta_{1}} u(\eta),
\end{array}\right.
$$

where $\alpha \in(1,2], \beta \in(3,4], \eta \in(0,1), b \in\left(0, \eta^{\frac{1-\alpha}{p-1}}\right)$, and $f \in C([0,1] \times$ $\left.[0,+\infty), \mathbf{R}^{+}\right)$. Existence of positive solutions is established by the upper and lower solutions method combined with the Schauder fixed point theorem.

In papers $[4,5]$, the case of a single equation is discussed in the $\phi$ Laplacian setting and existence of positive solutions were obtained, using fixed point arguments. More recently, the same method was employed by the authors of [13] to discuss the three-point BVP (1.3).

The main objective of this work is twofold: first, we extend bvp (1.2) to the case of the $\phi$-Laplacian operator and then we allow the nonlinearities $f, g$ to have space singularities (degenerate source terms). In addition, the latter functions obey some general condition that include classical superlinearities considered in above cited works. The existence of positive solutions is obtained here by introducing suitable positive cones in the space of continuous functions. The case where no singularity exists (regular case) is first discussed in Section 3. Then the singular cases are investigated in Sections 4 and 5, according to the number of singularities taken into account. For this purpose, a convenient approximating fixed point operator is introduced. It aims at approaching the singular problem by a sequence of regular ones and then to pass to the limit. Regarding the hypotheses, quite general growth conditions on the nonlinear functions $f$ and $g$ are supposed. We have adopted a fixed point method which turned out to be a powerful one to locate solutions since multiple positive solutions are obtained as well. Finally each existence theorem is illustrated by means of an example of application which exhibits some types of nonlinearities covered by our existence results.

\section{Preliminaries}

In this section, we collect some definitions and basic results used throughout. 


\subsection{Lemmas}

Definition 2.1. The Riemann-Liouville fractional integral of order $\alpha>0$ of a function $u: \mathbf{R}_{*}^{+} \rightarrow \mathbf{R}$ is defined by

$$
I_{0^{+}}^{\alpha} u(t)=\frac{1}{\Gamma(\alpha)} \int_{0}^{t}(t-s)^{\alpha-1} u(s) d s,
$$

provided that the right-hand side is pointwise defined on $\mathbf{R}_{*}^{+}$, where $\Gamma(\alpha)$ is the Euler gamma function defined by $\Gamma(\alpha)=\int_{0}^{+\infty} t^{\alpha-1} e^{-t} d t$.

Definition 2.2. The Riemann-Liouville fractional derivative of order $\alpha>$ 0 of a function $u: \mathbf{R}_{*}^{+} \rightarrow \mathbf{R}$ is defined by

$$
D_{0^{+}}^{\alpha} u(t)=\frac{d^{n}}{d t^{n}} I_{0^{+}}^{n-\alpha} u(t)=\frac{1}{\Gamma(n-\alpha)} \frac{d^{n}}{d t^{n}} \int_{0}^{t}(t-s)^{n-\alpha-1} u(s) d s,
$$

where $n$ is the smallest integer greater than or equal to $\alpha$, provided the right-hand side is pointwise defined on $\mathbf{R}_{*}^{+}$.

Lemma 2.1. Let $\alpha>0$. Then the following equality holds when $u \in$ $L(0,1)$ and $D_{0^{+}}^{\alpha} u(t) \in L(0,1)$ :

$$
I_{0^{+}}^{\alpha} D_{0^{+}}^{\alpha} u(t)=u(t)+c_{1} t^{\alpha-1}+c_{2} t^{\alpha-2}+\ldots+c_{n} t^{\alpha-n},
$$

where $c_{1}, c_{2}, \ldots, c_{n} \in \mathbf{R}$ and $n-1<\alpha \leq n$.

Definition 2.3. A nonempty subset $\mathcal{P}$ of a Banach space $E$ is called a cone if it is convex, closed, and satisfies the conditions:

(i) $\alpha x \in \mathcal{P}$, for all $x \in \mathcal{P}$ and $\alpha \geq 0$,

(ii) $x,-x \in \mathcal{P}$ implies that $x=0$.

Lemma 2.2. Let $\Omega$ be a bounded open set in a real Banach space $E, \mathcal{P}$ be a cone of $E$, and $A: \bar{\Omega} \cap \mathcal{P} \rightarrow \mathcal{P}$ be a completely continuous map. Suppose that $\lambda A x \neq x, \forall x \in \partial \Omega \cap \mathcal{P}, \lambda \in(0,1]$. Then $i(A, \Omega \cap \mathcal{P}, \mathcal{P})=1$.

Lemma 2.3. Let $\Omega$ be a bounded open set in a real Banach space $E, \mathcal{P}$ be a cone of $E$ and $A: \bar{\Omega} \cap \mathcal{P} \rightarrow \mathcal{P}$ be a completely continuous map. Suppose that $A x \not \leq x, \forall x \in \partial \Omega \cap \mathcal{P}$. Then $i(A, \Omega \cap \mathcal{P}, \mathcal{P})=0$. 
For more details on the theory and the computation of the fixed point index, we refer, e.g., to $[1,2,3]$.

In order to transform problem (1.1) into an equivalent integral equation, we make the change $\phi_{1}\left(D_{0^{+}}^{\beta_{1}} x(t)\right)=u(t)$ and then consider the bvp:

$$
\left\{\begin{array}{l}
-D_{0^{+}}^{\alpha_{1}} u(t)=v(t), t \in(0,1), \\
u(0)=0, u(1)=\phi_{1}\left(b_{1}\right) u\left(\mu_{1}\right)
\end{array}\right.
$$

whose solution is given by the follwong

Lemma 2.4. If $v \in C([0,1])$, then problem (2.1) has a unique solution

$$
u(t)=\int_{0}^{1} H_{1}(t, s) v(s) d s,
$$

where

$$
H_{1}(t, s)=h_{1}(t, s)+\frac{\phi_{1}\left(b_{1}\right) t^{\alpha_{1}-1}}{1-\phi_{1}\left(b_{1}\right) \mu_{1}^{\alpha_{1}-1}} h_{1}\left(\mu_{1}, s\right)
$$

and

$$
h_{1}(t, s)=\frac{1}{\Gamma\left(\alpha_{1}\right)} \begin{cases}(t(1-s))^{\alpha_{1}-1}, & 0 \leq t \leq s \leq 1 \\ (t(1-s))^{\alpha_{1}-1}-(t-s)^{\alpha_{1}-1}, & 0 \leq s \leq t \leq 1\end{cases}
$$

As a consequence

$$
\left\{\begin{array}{l}
-D_{0^{+}}^{\alpha_{1}}\left(\phi_{1}\left(D_{0^{+}}^{\beta_{1}} x(t)\right)=v(t), t \in(0,1)\right. \\
x(0)=x(1)=x^{\prime}(0)=x^{\prime}(1)=0 \\
D_{0^{+}}^{\beta_{1}} x(0)=0, D_{0^{+}}^{\beta_{1}} x(1)=b_{1} D_{0^{+}}^{\beta_{1}} x\left(\mu_{1}\right)
\end{array}\right.
$$

reads

$$
\left\{\begin{array}{l}
\left.D_{0^{+}}^{\beta_{1}} x(t)=\phi_{1}^{-1}\left(\int_{0}^{1} H_{1}(t, s) v(s)\right) d s\right), \quad t \in(0,1) \\
x(0)=x(1)=x^{\prime}(0)=x^{\prime}(1)=0 .
\end{array}\right.
$$

We have

Lemma 2.5. If $v \in C[0,1]$, then problem (2.2) has a unique solution given by

$$
x(t)=\int_{0}^{1} G_{1}(t, s) \phi_{1}^{-1}\left(\int_{0}^{1} H_{1}(s, \tau) v(\tau) d \tau\right) d s,
$$

where

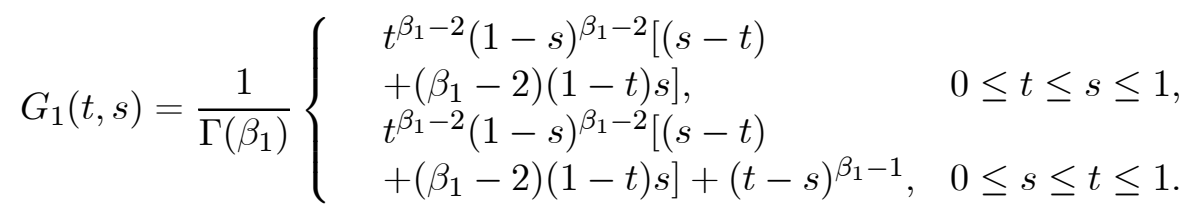


Proof. By Lemma 2.1

$$
-I_{0^{+}}^{\alpha_{1}} D_{0^{+}}^{\alpha_{1}} u(t)=I_{0^{+}}^{\alpha_{1}} v(t) .
$$

Then

$$
u(t)=-I_{0^{+}}^{\alpha_{1}} v(t)+c_{1} t^{\alpha_{1}-1}+c_{2} t^{\alpha_{1}-2}, c_{1}, c_{2} \in \mathbf{R} .
$$

The boundary conditions $u(0)=0$ and $u(1)=\phi_{1}\left(b_{1}\right) u\left(\mu_{1}\right)$ entail

$$
c_{1}=-\frac{\phi_{1}\left(b_{1}\right) I_{0^{+}}^{\alpha_{1}} v\left(\mu_{1}\right)-I_{0^{+}}^{\alpha_{1}} v(1)}{1-\phi_{1}\left(b_{1}\right) \mu_{1}^{\alpha_{1}-1}} \text { and } c_{2}=0 .
$$

Hence the solution $u=u(t)$ of problem (2.1) satisfies

$$
\begin{aligned}
u(t) & =-I_{0^{+}}^{\alpha_{1}} v(t)+\frac{t^{\alpha_{1}-1}}{1-\phi_{1}\left(b_{1}\right) \mu_{1}^{\alpha_{1}-1}} I_{0^{+}}^{\alpha_{1}} v(1)-\frac{\phi_{1}\left(b_{1}\right) t^{\alpha_{1}-1}}{1-\phi_{1}\left(b_{1}\right) \mu_{1}^{\alpha_{1}-1}} I_{0^{+}}^{\alpha_{1}} v\left(\mu_{1}\right) \\
& =-I_{0^{+}}^{\alpha_{1}} v(t)+\frac{t^{\alpha_{1}-1}}{1-\phi_{1}\left(b_{1}\right) \mu_{1}^{\alpha_{1}-1}} I_{0^{+}}^{\alpha_{1}} v(1)-\frac{\phi_{1}\left(b_{1}\right) t^{\alpha_{1}-1}}{1-\phi_{1}\left(b_{1}\right) \mu_{1}^{\alpha_{1}-1}} I_{0^{+}}^{\alpha_{1}} v\left(\mu_{1}\right) \\
& +t^{\alpha_{1}-1} I_{0^{+}}^{\alpha_{1}} v(1)-t^{\alpha_{1}-1} I_{0^{+}}^{\alpha_{1}} v(1) \\
& =t^{\alpha_{1}-1} I_{0^{+}}^{\alpha_{1}} v(1)-I_{0^{+}}^{\alpha_{1}} v(t)+\frac{\phi_{1}\left(b_{1}\right) t^{\alpha_{1}-1}}{1-\phi_{1}\left(b_{1}\right) \mu_{1}^{\alpha_{1}-1}}\left(\mu_{1}^{\alpha_{1}-1} I_{0^{+}}^{\alpha_{1}} v(1)-I_{0^{+}}^{\alpha_{1}} v\left(\mu_{1}\right)\right) \\
& =\int_{0}^{1} h_{1}(t, s) v(s) d s+\frac{\phi_{1}\left(b_{1}\right) t^{\alpha_{1}-1}}{1-\phi_{1}\left(b_{1}\right) \mu_{1}^{\alpha_{1}-1}} \int_{0}^{1} h_{1}\left(\mu_{1}, s\right) v(s) d s .
\end{aligned}
$$

Similarly by Lemma 2.1, we have

$$
\left.-I_{0^{+}}^{\beta_{1}} D_{0^{+}}^{\beta_{1}} x(t)=I_{0^{+}}^{\beta_{1}} \phi_{1}^{-1}\left(\int_{0}^{1} H_{1}(t, s) v(s)\right) d s\right) .
$$

Then

$\left.x(t)=-I_{0^{+}}^{\beta_{1}} \phi_{1}^{-1}\left(\int_{0}^{1} H_{1}(t, s) v(s)\right) d s\right)+c_{1} t^{\beta_{1}-1}+c_{2} t^{\beta_{1}-2}+c_{3} t^{\beta_{1}-3}+c_{4} t^{\beta_{1}-4}$,

where $c_{1}, c_{2}, c_{3}, c_{4} \in \mathbf{R}$. Applying the boundary conditions $x(0)=x(1)=$ $x^{\prime}(0)=x^{\prime}(1)=0$, we get

$$
\begin{aligned}
c_{1}= & -\frac{1}{\Gamma\left(\beta_{1}\right)} \int_{0}^{1}(1-s)^{\beta_{1}-1} \phi_{1}^{-1}\left(\int_{0}^{1} H_{1}(s, \tau) v(\tau) d \tau\right) d s \\
& -\frac{\beta_{1}-1}{\Gamma\left(\beta_{1}\right)} \int_{0}^{1}(1-s)^{\beta_{1}-2} s \phi_{1}^{-1}\left(\int_{0}^{1} H_{1}(s, \tau) v(\tau) d \tau\right) d s \\
c_{2}= & \frac{\beta_{1}-1}{\Gamma\left(\beta_{1}\right)} \int_{0}^{1}(1-s)^{\beta_{1}-2} s \phi_{1}^{-1}\left(\int_{0}^{1} H_{1}(s, \tau) v(\tau) d \tau\right) d s, \\
c_{3}=c_{4}= & 0 .
\end{aligned}
$$


Therefore, the solution $x(t)$ of problem (2.2) is given by

$$
\begin{aligned}
x(t)= & \frac{1}{\Gamma\left(\beta_{1}\right)} \int_{0}^{t}(t-s)^{\beta_{1}-1} \phi_{1}^{-1}\left(\int_{0}^{1} H_{1}(s, \tau) v(\tau) d \tau\right) d s \\
& -\frac{t^{\beta_{1}-1}}{\Gamma\left(\beta_{1}\right)} \int_{0}^{1}(1-s)^{\beta_{1}-1} \phi_{1}^{-1}\left(\int_{0}^{1} H_{1}(s, \tau) v(\tau) d \tau\right) d s \\
& -\frac{t^{\beta_{1}-1}\left(\beta_{1}-1\right)}{\Gamma\left(\beta_{1}\right)} \int_{0}^{1}(1-s)^{\beta_{1}-2} s \phi_{1}^{-1}\left(\int_{0}^{1} H_{1}(s, \tau) v(\tau) d \tau\right) d s \\
& +\frac{t^{\beta_{1}-2}\left(\beta_{1}-1\right)}{\Gamma\left(\beta_{1}\right)} \int_{0}^{1}(1-s)^{\beta_{1}-2} s \phi_{1}^{-1}\left(\int_{0}^{1} H_{1}(s, \tau) v(\tau) d \tau\right) d s \\
= & \frac{1}{\Gamma\left(\beta_{1}\right)} \int_{0}^{t}(t-s)^{\beta_{1}-1} \phi_{1}^{-1}\left(\int_{0}^{1} H_{1}(s, \tau) v(\tau) d \tau\right) d s \\
& +\frac{t^{\beta_{1}-2}(1-s)^{\beta_{1}-2}}{\Gamma\left(\beta_{1}\right)} \int_{0}^{1}\left[\left(\beta_{1}-1\right) s(1-t)-t(1-s)\right] \phi_{1}^{-1}\left(\int_{0}^{1} H_{1}(s, \tau) v(\tau) d \tau\right) d s \\
= & \frac{1}{\Gamma\left(\beta_{1}\right)} \int_{0}^{t}(t-s)^{\beta_{1}-1} \phi_{1}^{-1}\left(\int_{0}^{1} H_{1}(s, \tau) v(\tau) d \tau\right) d s \\
& +\frac{t^{\beta_{1}-2}(1-s)^{\beta_{1}-2}}{\Gamma\left(\beta_{1}\right)} \int_{0}^{1}\left[(s-t)+\left(\beta_{1}-2\right)(1-t) s\right] \phi_{1}^{-1}\left(\int_{0}^{1} H_{1}(s, \tau) v(\tau) d \tau\right) d s \\
= & \int_{0}^{1} G_{1}(t, s) \phi_{1}^{-1}\left(\int_{0}^{1} H_{1}(s, \tau) v(\tau) d \tau\right) d s .
\end{aligned}
$$

Lemma 2.6. The functions $G_{1}(t, s), H_{1}(t, s)$ enjoy the following properties:

(a) $G_{1}, H_{1}$ are continuous on $[0,1] \times[0,1]$,

(b) $\frac{\left(\beta_{1}-2\right) K_{1}(t) L_{1}(s)}{\Gamma\left(\beta_{1}\right)} \leq G_{1}(t, s) \leq \frac{M_{1}}{\Gamma\left(\beta_{1}\right)}, \forall(t, s) \in(0,1) \times(0,1)$, where

$$
\begin{aligned}
& K_{1}(t)=t^{\beta_{1}-2}(1-t)^{2}, L_{1}(s)=s^{2}(1-s)^{\beta_{1}-2}, M_{1}=\max \left\{\beta_{1}-1,\left(\beta_{1}-2\right)^{2}\right\} \\
& \text { (c) } G_{1}(t, s) \geq \frac{\beta_{1}-2}{M_{1}} t^{2}(1-t)^{2} \sup _{t \in[0,1]} G_{1}(t, s), \forall(t, s) \in(0,1) \times(0,1), \\
& \text { (d) } H_{1}(t, s) \leq \lambda_{1}(1-s)^{\alpha_{1}-1}, \forall(t, s) \in(0,1) \times(0,1), \text { where }
\end{aligned}
$$

$$
\lambda_{1}=\frac{1}{\left[1-\phi_{1}\left(b_{1}\right) \mu_{1}^{\alpha_{1}-1}\right] \Gamma\left(\alpha_{1}\right)} .
$$

Proof. (b) In [6], the authors proved that

$$
\left(\beta_{1}-2\right) K_{1}(t) L_{1}(s) \leq \Gamma\left(\beta_{1}\right) G_{1}(t, s) \leq M_{1} L_{1}(s), \forall(t, s) \in(0,1) \times(0,1) .
$$


(c) Let $t \in(0,1)$, we have

$$
\begin{aligned}
G_{1}(t, s) & \geq \frac{\left(\beta_{1}-2\right) K_{1}(t) L_{1}(s)}{\Gamma\left(\beta_{1}\right)} \\
& \geq \frac{\left(\beta_{1}-2\right) K_{1}(t) M_{1} L_{1}(s)}{M_{1} \Gamma\left(\beta_{1}\right)} \\
& \geq \frac{\left(\beta_{1}-2\right) t^{2}(1-t)^{2} M_{1} L_{1}(s)}{M_{1} \Gamma\left(\beta_{1}\right)} .
\end{aligned}
$$

Furthermore,

$$
\sup _{t \in[0,1]} G_{1}(t, s) \leq \frac{M_{1} L_{1}(s)}{\Gamma\left(\beta_{1}\right)}
$$

whence comes the result.

(d) We have

$$
h_{1}(t, s) \leq \frac{1}{\Gamma\left(\alpha_{1}\right)}[t(1-s)]^{\alpha_{1}-1} \leq \frac{(1-s)^{\alpha_{1}-1}}{\Gamma\left(\alpha_{1}\right)} .
$$

Then

$$
\begin{aligned}
H_{1}(t, s) & \leq \frac{(1-s)^{\alpha_{1}-1}}{\Gamma\left(\alpha_{1}\right)}+\frac{\phi_{1}\left(b_{1}\right)}{1-\phi_{1}(b) \mu_{1}^{\alpha_{1}-1}} \frac{\mu_{1}^{\alpha_{1}-1}(1-s)^{\alpha_{1}-1}}{\Gamma\left(\alpha_{1}\right)} \\
& \leq \frac{1}{1-\phi_{1}\left(b_{1}\right) \mu_{1}^{\alpha_{1}-1}} \frac{(1-s)^{\alpha_{1}-1}}{\Gamma\left(\alpha_{1}\right)} \\
& \leq \lambda_{1}(1-s)^{\alpha_{1}-1} .
\end{aligned}
$$

Similar results are obtained for the following BVP, by replacing $\alpha_{1}$ by $\alpha_{2}$ and $\beta_{1}$ by $\beta_{2}$ :

$$
\left\{\begin{array}{l}
-D_{0^{+}}^{\alpha_{2}}\left(\phi_{2}\left(D_{0^{+}}^{\beta_{2}} y(t)\right)=v(t), t \in(0,1)\right. \\
y(0)=y(1)=y^{\prime}(0)=y^{\prime}(1)=0, \\
D_{0^{+}}^{\beta_{2}} y(0)=0, D_{0^{+}}^{\beta_{2}} y(1)=b_{2} D_{0^{+}}^{\beta_{2}} y\left(\mu_{2}\right) .
\end{array}\right.
$$

To complete the functional setting, we consider the Banach space $E=$ $\{(x, y) \in C([0,1] \times[0,1])\}$ with the norm $\|(x, y)\|=\|x\|+\|y\|$, where $\|x\|=\sup _{t \in[0,1]}|x(t)|$. Finally, let the cone $\mathcal{P}$ be given by

$\mathcal{P}=\left\{(x, y) \in E: x(t) \geq \frac{\beta_{1}-2}{M_{1}} \gamma(t)\|x\|, y(t) \geq \frac{\beta_{2}-2}{M_{2}} \gamma(t)\|y\|, \quad \forall t \in[0,1]\right\}$,

where $\gamma(t)=t^{2}(1-t)^{2}, t \in[0,1]$. 


\section{Regular nonlinearities}

In this section, we suppose that the functions $f, g:[0,1] \times \mathbf{R}^{+} \times \mathbf{R}^{+} \rightarrow \mathbf{R}^{+}$ are continuous and satisfy

$\left(\mathcal{H}_{\mathbf{1}}\right)$ There exist nonegative real-valued functions $m_{i} \in L([0,1])$ and $\psi_{i} \in$ $C\left(\mathbf{R}^{+} \times \mathbf{R}^{+}, \mathbf{R}^{+}\right)$such that $\psi_{i}$ are nondecreasing functions in each argument (for $i \in\{0,1\}$ ), with

$$
\begin{aligned}
& f(t, x, y) \leq m_{1}(t) \psi_{1}(x, y), \forall(t, x, y) \in[0,1] \times\left(\mathbf{R}^{+}\right)^{2} \\
& g(t, x, y) \leq m_{2}(t) \psi_{2}(x, y), \forall(t, x, y) \in[0,1] \times\left(\mathbf{R}^{+}\right)^{2}
\end{aligned}
$$

with

$$
\int_{0}^{1}(1-\tau)^{\alpha_{i}-1} a_{i}(\tau) m_{i}(\tau) d \tau<+\infty, \quad i \in\{0,1\}
$$

$\left(\mathcal{H}_{\mathbf{2}}\right)$

$$
\sup _{c>0} \frac{c}{\sum_{i=1}^{2} \frac{M_{i}}{\Gamma\left(\beta_{i}\right)} \phi_{i}^{-1}\left(\lambda_{i} \psi_{i}(c, c) \int_{0}^{1}(1-\tau)^{\alpha_{i}-1} a_{i}(\tau) m_{i}(\tau) d \tau\right)}>1 .
$$

$\left(\mathcal{H}_{3}\right)$

There exists $\sigma \in\left(0, \frac{1}{2}\right)$ such that

$$
\lim _{x+y \rightarrow+\infty} \inf _{t \in[\sigma, 1-\sigma]} \frac{f(t, x, y)}{\phi_{1}(x+y)} \geq l_{1} \text { and } \lim _{x+y \rightarrow+\infty} \inf _{t \in[\sigma, 1-\sigma]} \frac{g(t, x, y)}{\phi_{2}(x+y)} \geq l_{2},
$$

where

$$
l_{i}=1+\frac{\phi_{i}\left(\frac{\Gamma\left(\beta_{i}\right)}{2 c_{i}(1-2 \sigma)^{2}\left(\beta_{i}-2\right)}\right)}{\int_{\sigma}^{1-\sigma} a_{i}(\tau) H_{i}\left(d_{i}, \tau\right) d \tau}, i \in\{1,2\},
$$

such that

$c_{i}=\min _{(t, s) \in([\sigma, 1-\sigma])^{2}} K_{i}(t) L_{i}(s), H_{i}\left(d_{i}, \tau\right)=\min _{s \in[\sigma, 1-\sigma]} H_{i}(s, \tau), \min _{t \in[\sigma, 1-\sigma]} a_{i}(\tau) \neq 0$.

$\left(\mathcal{H}_{4}\right)$

$$
\lim _{x+y \rightarrow 0} \inf _{t \in[\sigma, 1-\sigma]} \frac{f(t, x, y)}{\phi_{1}(x+y)} \geq N_{1} \text { and } \lim _{x+y \rightarrow 0} \inf _{t \in[\sigma, 1-\sigma]} \frac{g(t, x, y)}{\phi_{2}(x+y)} \geq N_{2},
$$


where

$$
N_{i}=1+\frac{\phi_{i}\left(\frac{\Gamma\left(\beta_{i}\right)}{2 N c_{i}(1-2 \sigma)^{2}\left(\beta_{i}-2\right)}\right)}{\int_{\sigma}^{1-\sigma} a_{i}(\tau) \phi_{i}(\gamma(\tau)) H_{i}\left(d_{i}, \tau\right) d \tau}, \quad i \in\{1,2\} .
$$

Let $N=\max \left\{\frac{\beta_{1}-2}{M_{1}}, \frac{\beta_{2}-2}{M_{2}}\right\}$.

For $(x, y) \in \mathcal{P}$, define operators $A, B: E \longrightarrow C([0,1])$ by

$$
\begin{aligned}
& A(x, y)(t)=\int_{0}^{1} G_{1}(t, s) \phi_{1}^{-1}\left(\int_{0}^{1} H_{1}(s, \tau) a_{1}(\tau) f(\tau, x(\tau), y(\tau)) d \tau\right) d s \\
& B(x, y)(t)=\int_{0}^{1} G_{2}(t, s) \phi_{2}^{-1}\left(\int_{0}^{1} H_{2}(s, \tau) a_{2}(\tau) g(\tau, x(\tau), y(\tau)) d \tau\right) d s
\end{aligned}
$$

and then

$$
T(x, y)=(A(x, y), B(x, y)) .
$$

Proposition 3.1. Assume that condition $\left(\mathcal{H}_{1}\right)$ holds. Then $T: \mathcal{P} \longrightarrow \mathcal{P}$ is a completely continuous operator.

Proof. Step 1: We prove that for $(x, y) \in \mathcal{P}, T(x, y) \in \mathcal{P}$. We have $A(x, y)(t) \geq 0, B(x, y)(t) \geq 0 ; \forall t \in[0,1]$, and by $(c)$ of Lemma 2.6

$$
\begin{aligned}
A(x, y)(t) & =\int_{0}^{1} G_{1}(t, s) \phi_{1}^{-1}\left(\int_{0}^{1} H_{1}(s, \tau) a_{1}(\tau) f(\tau, x(\tau), y(\tau)) d \tau\right) d s \\
& \geq \frac{\beta_{1}-2}{M_{1}} \gamma(t) \int_{0}^{1} \sup _{t \in[0,1]} G_{1}(t, s) \phi_{1}^{-1}\left(\int_{0}^{1} H_{1}(s, \tau) a_{1}(\tau) f(\tau, x(\tau), y(\tau)) d \tau\right) d s \\
& \geq \frac{\beta_{1}-2}{M_{1}} \gamma(t) \sup _{t \in[0,1]} \int_{0}^{1} G_{1}(t, s) \phi_{1}^{-1}\left(\int_{0}^{1} H_{1}(s, \tau) a_{1}(\tau) f(\tau, x(\tau), y(\tau)) d \tau\right) d s \\
& \geq \frac{\beta_{1}-2}{M_{1}} \gamma(t)\|A(x, y)\| .
\end{aligned}
$$

Similarly, by $(c)$ of Lemma 2.6, we have

$$
\begin{aligned}
B(x, y)(t) & =\int_{0}^{1} G_{2}(t, s) \phi_{2}^{-1}\left(\int_{0}^{1} H_{2}(s, \tau) a_{2}(\tau) g(\tau, x(\tau), y(\tau)) d \tau\right) d s \\
& \geq \frac{\beta_{2}-2}{M_{2}} \gamma(t) \int_{0}^{1} \sup _{t \in[0,1]} G_{2}(t, s) \phi_{2}^{-1}\left(\int_{0}^{1} H_{2}(s, \tau) a_{2}(\tau) g(\tau, x(\tau), y(\tau)) d \tau\right) d s \\
& \geq \frac{\beta_{2}-2}{M_{2}} \gamma(t) \sup _{t \in[0,1]} \int_{0}^{1} G_{2}(t, s) \phi_{2}^{-1}\left(\int_{0}^{1} H_{2}(s, \tau) a_{2}(\tau) g(\tau, x(\tau), y(\tau)) d \tau\right) d s \\
& \geq \frac{\beta_{2}-2}{M_{2}} \gamma(t)\|B(x, y)\| .
\end{aligned}
$$

Hence $T(\mathcal{P}) \subset \mathcal{P}$.

Step 2: Let $D$ is a bounded set; then there exists $r>0$ such that

$$
\forall(x, y) \in D,\|(x, y)\| \leq r .
$$


By $\left(\mathcal{H}_{1}\right)$ and the properties $(b)$ and $(d)$ of Lemma 2.6, we have

$$
\begin{aligned}
\|A(x, y)\| & =\left\|\int_{0}^{1} G_{1}(t, s) \phi_{1}^{-1}\left(\int_{0}^{1} H_{1}(s, \tau) a_{1}(\tau) f(\tau, x(\tau), y(\tau)) d \tau\right) d s\right\| \\
& \leq \frac{M_{1}}{\Gamma\left(\beta_{1}\right)} \int_{0}^{1} \phi_{1}^{-1}\left(\int_{0}^{1} H_{1}(s, \tau) a_{1}(\tau) f(\tau, x(\tau), y(\tau)) d \tau\right) d s \\
& \leq \frac{M_{1}}{\Gamma\left(\beta_{1}\right)} \phi_{1}^{-1}\left(\int_{0}^{1} \lambda_{1}(1-\tau)^{\alpha_{1}-1} a_{1}(\tau) m_{1}(\tau) \psi_{1}(x(\tau), y(\tau))\right) d \tau \\
& \leq \frac{M_{1}}{\Gamma\left(\beta_{1}\right)} \phi_{1}^{-1}\left(\lambda_{1} \psi_{1}(r, r) \int_{0}^{1}(1-\tau)^{\alpha_{1}-1} a_{1}(\tau) m_{1}(\tau)\right) d \tau<+\infty .
\end{aligned}
$$

Similarly by $\left(\mathcal{H}_{1}\right)$ and the properties $(b)$ and $(d)$ of Lemma 2.6 , we have

$$
\|B(x, y)\| \leq \frac{M_{2}}{\Gamma\left(\beta_{2}\right)} \phi_{2}^{-1}\left(\lambda_{2} \psi_{2}(r, r) \int_{0}^{1}(1-\tau)^{\alpha_{2}-1} a_{2}(\tau) m_{2}(\tau)\right) d \tau<+\infty .
$$

Step 3: Given $t, t^{\prime} \in[0,1]\left(t<t^{\prime}\right)$, we have

$$
\begin{aligned}
& \left|A(x, y)(t)-A(x, y)\left(t^{\prime}\right)\right| \\
\leq & \int_{0}^{1}\left|G_{1}(t, s)-G_{1}\left(t^{\prime}, s\right)\right| \phi_{1}^{-1}\left(\int_{0}^{1} a_{1}(\tau) H_{1}(s, \tau) f(\tau, x(\tau), y(\tau)) d \tau\right) d s .
\end{aligned}
$$

Similarly,

$$
\begin{aligned}
& \left|B(x, y)(t)-B(x, y)\left(t^{\prime}\right)\right| \\
\leq & \int_{0}^{1}\left|G_{2}(t, s)-G_{2}\left(t^{\prime}, s\right)\right| \phi_{2}^{-1}\left(\int_{0}^{1} a_{2}(\tau) H_{2}(s, \tau) g(\tau, x(\tau), y(\tau)) d \tau\right) d s .
\end{aligned}
$$

By the continuity of $G_{1}, G_{2}$, and appealing to Arzela-Ascoli Lemma, we deduce that $T$ is completely continuous.

Theorem 3.1. Assume that conditions $\left(\mathcal{H}_{1}\right)-\left(\mathcal{H}_{4}\right)$ hold. Then bvp (1.1) has at least two positive solutions.

Proof. From condition $\left(\mathcal{H}_{2}\right)$, there exists $R_{2}>0$, such that

$$
\frac{R_{2}}{\sum_{i=1}^{2} \frac{M_{i}}{\Gamma\left(\beta_{i}\right)} \phi_{i}^{-1}\left(\lambda_{i} \psi_{i}\left(R_{2}, R_{2}\right) \int_{0}^{1}(1-\tau)^{\alpha_{i}-1} a_{i}(\tau) m_{i}(\tau) d \tau\right)}>1 .
$$

And from condition $\left(\mathcal{H}_{4}\right)$ there exists $r>0$ with $r<R_{2}$ such that $f(t, x, y) \geq N_{1} \phi_{1}(x+y)$ with $x+y \leq r$, for all $t \in[\sigma, 1-\sigma]$ and there exists $r^{\prime}>0$ such that $r^{\prime}<R_{2}$ and $g(t, x, y) \geq N_{2} \phi_{2}(x+y)$ with $x+y \leq r^{\prime}$, for all $t \in[\sigma, 1-\sigma]$.

Step 1. Define the open ball

$$
\Omega_{1}=\left\{(x, y) \in E:\|(x, y)\| \leq R_{1}\right\}
$$


where $R_{1}=\min \left\{r, r^{\prime}\right\}$. We show that $T(x, y) \not \subset(x, y)$, for all $(x, y) \in$ $\partial \Omega_{1} \cap \mathcal{P}$. Suppose on the contrary that there exists $\left(x_{0}, y_{0}\right) \in \partial \Omega_{1} \cap \mathcal{P}$ such that $T\left(x_{0}, y_{0}\right) \leq\left(x_{0}, y_{0}\right)$. By $\left(\mathcal{H}_{4}\right)$ and $(b)$ of Lemma 2.6, we have the estimates

$$
\begin{aligned}
x_{0}(t) & \geq A\left(x_{0}(t), y_{0}(t)\right) \\
& \geq \frac{\beta_{1}-2}{\Gamma\left(\beta_{1}\right)} K_{1}(t) \int_{\sigma}^{1-\sigma} L_{1}(s) \phi_{1}^{-1}\left(\int_{\sigma}^{1-\sigma} a_{1}(\tau) H_{1}(s, \tau) \phi_{1}\left(x_{0}(\tau)+y_{0}(\tau)\right) N_{1} d \tau\right) d s \\
& \geq N R_{1} \frac{\beta_{1}-2}{\Gamma\left(\beta_{1}\right)} K_{1}(t) \int_{\sigma}^{1-\sigma} L_{1}(s) \phi_{1}^{-1}\left(\int_{\sigma}^{1-\sigma} a_{1}(\tau) \phi_{1}(\gamma(\tau)) H_{1}\left(d_{1}, \tau\right) N_{1} d \tau\right) d s \\
& \geq N R_{1} \frac{\beta_{1}-2}{\Gamma\left(\beta_{1}\right)} K_{1}(t) \int_{\sigma}^{1-\sigma} L_{1}(s) \phi_{1}^{-1}\left(\int_{\sigma}^{1-\sigma} a_{1}(\tau) \phi_{1}(\gamma(\tau)) H_{1}\left(d_{1}, \tau\right)\right. \\
& \left.\left.+\phi_{1}\left(\frac{\Gamma\left(\beta_{1}\right)}{2 N c_{1}(1-2 \sigma)^{2}\left(\beta_{1}-2\right)}\right)\right) d \tau\right) d s \\
& \left.>N R_{1} \frac{\beta_{1}-2}{\Gamma\left(\beta_{1}\right)} K_{1}(t) \int_{\sigma}^{1-\sigma} L_{1}(s) \phi_{1}^{-1}\left(\int_{\sigma}^{1-\sigma} \phi_{1}\left(\frac{\Gamma\left(\beta_{1}\right)}{2 N c_{1}(1-2 \sigma)^{2}\left(\beta_{1}-2\right)}\right)\right) d \tau\right) d s \\
& >\frac{R_{1}}{2} .
\end{aligned}
$$

Similarly, by $\left(\mathcal{H}_{4}\right)$ and $(b)$ of Lemma 2.6 , we get

$$
\begin{aligned}
y_{0}(t) & \geq B\left(x_{0}(t), y_{0}(t)\right) \\
\geq & \frac{\beta_{2}-2}{\Gamma\left(\beta_{2}\right)} K_{2}(t) \int_{\sigma}^{1-\sigma} L_{2}(s) \phi_{2}^{-1}\left(\int_{\sigma}^{1-\sigma} a_{2}(\tau) H_{2}(s, \tau) \phi_{2}\left(x_{0}(\tau)+y_{0}(\tau) N_{2} d \tau\right) d s\right. \\
& \geq N R_{1} \frac{\beta_{2}-2}{\Gamma\left(\beta_{2}\right)} K_{2}(t) \int_{\sigma}^{1-\sigma} L_{2}(s) \phi_{2}^{-1}\left(\int_{\sigma}^{1-\sigma} a_{2}(\tau) \phi_{2}(\gamma(\tau)) H_{2}\left(d_{2}, \tau\right) N_{2} d \tau\right) d s . \\
& \geq N R_{1} \frac{\beta_{2}-2}{\Gamma\left(\beta_{1}\right)} K_{2}(t) \int_{\sigma}^{1-\sigma} L_{2}(s) \phi_{2}^{-1}\left(\int_{\sigma}^{1-\sigma} a_{2}(\tau) \phi_{2}(\gamma(\tau)) H_{2}\left(d_{2}, \tau\right)\right. \\
& \left.\left.+\phi_{2}\left(\frac{\Gamma\left(\beta_{2}\right)}{2 N c_{2}(1-2 \sigma)^{2}\left(\beta_{2}-2\right)}\right)\right) d \tau\right) d s \\
& \left.>N R_{1} \frac{\beta_{2}-2}{\Gamma\left(\beta_{2}\right)} K_{2}(t) \int_{\sigma}^{1-\sigma} L_{2}(s) \phi_{2}^{-1}\left(\int_{\sigma}^{1-\sigma} \phi_{2}\left(\frac{\Gamma\left(\beta_{1}\right)}{2 N c_{2}(1-2 \sigma)^{2}\left(\beta_{2}-2\right)}\right)\right) d \tau\right) d s \\
& >\frac{R_{1}}{2} .
\end{aligned}
$$

Hence

$$
\left\|\left(x_{0}, y_{0}\right)\right\|>R_{1},
$$

contradicting $\left\|\left(x_{0}, y_{0}\right)\right\|=R_{1}$. Using Lemma 2.3, we conclude that

$$
i\left(T, \Omega_{1} \cap \mathcal{P}, \mathcal{P}\right)=0 .
$$

Step 2. Let

$$
\Omega_{2}=\left\{(x, y) \in E:\|(x, y)\|<R_{2}\right\} .
$$

We claim that $(x, y) \neq \lambda T(x, y)$ for all $(x, y) \in \partial \Omega_{2} \cap \mathcal{P}$ and $\lambda \in(0,1]$. On the contrary, suppose that there exist $\left(x_{0}, y_{0}\right) \in \partial \Omega_{2} \cap \mathcal{P}$ and $\lambda_{0} \in(0,1]$ such that $\left(x_{0}, y_{0}\right)=\lambda_{0} T\left(x_{0}, y_{0}\right)$. Then

$$
x_{0}=\lambda_{0} A\left(x_{0}, y_{0}\right) \text { and } y_{0}=\lambda_{0} B\left(x_{0}, y_{0}\right) .
$$


By $\left(\mathcal{H}_{1}\right)$ and the properties $(b)$ and $(d)$ of Lemma 2.6, we have

$$
\begin{aligned}
\left\|x_{0}\right\| & =\left\|\lambda_{0} A\left(x_{0}, y_{0}\right)\right\| \\
& =\lambda_{0}\left\|\int_{0}^{1} G_{1}(t, s) \phi_{1}^{-1}\left(\int_{0}^{1} H_{1}(s, \tau) a_{1}(\tau) f\left(\tau, x_{0}(\tau), y_{0}(\tau)\right) d \tau\right) d s\right\| \\
& \leq \lambda_{0} \frac{M_{1}}{\Gamma\left(\beta_{1}\right)} \phi_{1}^{-1}\left(\int_{0}^{1} a_{1}(\tau) m_{1}(\tau) \lambda_{1}(1-\tau)^{\alpha_{1}-1} \psi_{1}\left(x_{0}(\tau), y_{0}(\tau)\right) d \tau\right) \\
& \leq \frac{M_{1}}{\Gamma\left(\beta_{1}\right)} \phi_{1}^{-1}\left(\lambda_{1} \int_{0}^{1} a_{1}(\tau) m_{1}(\tau)(1-\tau)^{\alpha_{1}-1} \psi_{1}\left(R_{2}, R_{2}\right) d \tau\right) \\
& \leq \frac{M_{1}}{\Gamma\left(\beta_{1}\right)} \phi_{1}^{-1}\left(\lambda_{1} \psi_{1}\left(R_{2}, R_{2}\right) \int_{0}^{1} a_{1}(\tau) m_{1}(\tau)(1-\tau)^{\alpha_{1}-1} d \tau\right)
\end{aligned}
$$

and

$$
\begin{aligned}
\left\|y_{0}\right\| & =\left\|\lambda_{0} B\left(x_{0}, y_{0}\right)\right\| \\
& =\lambda_{0}\left\|\int_{0}^{1} G_{2}(t, s) \phi_{2}^{-1}\left(\int_{0}^{1} H_{2}(s, \tau) a_{2}(\tau) g\left(\tau, x_{0}(\tau), y_{0}(\tau)\right) d \tau\right) d s\right\| \\
& \leq \lambda_{0} \frac{M_{2}}{\Gamma\left(\beta_{2}\right)} \phi_{2}^{-1}\left(\int_{0}^{1} a_{2}(\tau) m_{2}(\tau) \lambda_{2}(1-\tau)^{\alpha_{2}-1} \psi_{2}\left(x_{0}(\tau), y_{0}(\tau)\right) d \tau\right) \\
& \leq \frac{M_{2}}{\Gamma\left(\beta_{2}\right)} \phi_{2}^{-1}\left(\lambda_{2} \int_{0}^{1} a_{2}(\tau) m_{2}(\tau)_{2}(1-\tau)^{\alpha_{2}-1} \psi_{2}\left(R_{2}, R_{2}\right) d \tau\right) \\
& \leq \frac{M_{2}}{\Gamma\left(\beta_{2}\right)} \phi_{2}^{-1}\left(\lambda_{2} \psi_{2}\left(R_{2}, R_{2}\right) \int_{0}^{1} a_{2}(\tau) m_{2}(\tau)(1-\tau)^{\alpha_{2}-1} d \tau\right) .
\end{aligned}
$$

We conclude that

$$
\begin{aligned}
R_{2}=\left\|\left(x_{0}, y_{0}\right)\right\| \leq & \frac{M_{1}}{\Gamma\left(\beta_{1}\right)} \phi_{1}^{-1}\left(\lambda_{1} \psi_{1}\left(R_{2}, R_{2}\right) \int_{0}^{1} a_{1}(\tau) m_{1}(\tau)(1-\tau)^{\alpha_{1}-1} d \tau\right) \\
& +\frac{M_{2}}{\Gamma\left(\beta_{2}\right)} \phi_{2}^{-1}\left(\lambda_{2} \psi_{2}\left(R_{2}, R_{2}\right) \int_{0}^{1} a_{2}(\tau) m_{2}(\tau)(1-\tau)^{\alpha_{2}-1} d \tau\right),
\end{aligned}
$$

which is a contradiction to (3.3). Using Lemma 2.2, we deduce that

$$
i\left(A, \Omega_{2} \cap \mathcal{P}, \mathcal{P}\right)=1
$$

Therefore

$$
i\left(T,\left(\Omega_{2} \backslash \overline{\Omega_{1}}\right) \cap \mathcal{P}, \mathcal{P}\right)=1 .
$$

Then $T$ has a least one fixed point $\left(x_{0}, y_{0}\right)$ such that $R_{1}<\left\|\left(x_{0}, y_{0}\right)\right\|<R_{2}$.

Step 3. From $\left(\mathcal{H}_{3}\right)$, there exists $R>0$ such that $R>R_{2}$ and $f(t, x, y) \geq$ $l_{1} \phi_{1}(x+y)$ with $x+y \geq R$, for all $t \in[\sigma, 1-\sigma]$ and there exists $R^{\prime}>0$ such that $R^{\prime}>R_{2}$ and $g(t, x, y) \geq l_{2} \phi_{2}(x+y)$ with $x+y \geq R^{\prime}$, for all $t \in[\sigma, 1-\sigma]$. Define the open ball

$$
\Omega_{3}=\left\{(x, y) \in E:\|(x, y)\| \leq R_{3}\right\},
$$

where $R_{3}=\max \left\{R, R^{\prime}\right\}$. To show that $T(x, y)(x, y)$ for all $(x, y) \subset \partial \Omega_{3} \cap \mathcal{P}$, suppose on the contrary that there exists $\left(x_{1}, y_{1}\right) \in \partial \Omega_{3} \cap \mathcal{P}$ such that 
$T\left(x_{1}, y_{1}\right) \leq\left(x_{1}, y_{1}\right)$. Using $\left(\mathcal{H}_{3}\right)$ and $(b)$ of Lemma 2.6, we find

$$
\begin{aligned}
x_{1}(t) \geq & A\left(x_{1}(t), y_{1}(t)\right) \\
\geq & \frac{\beta_{1}-2}{\Gamma\left(\beta_{1}\right.} K_{1}(t) \int_{\sigma}^{1-\sigma} L_{1}(s) \phi_{1}^{-1}\left(\int_{0}^{1} a_{1}(\tau) H_{1}(s, \tau) \phi_{1}\left(x_{1}(\tau)+y_{1}(\tau)\right) l_{1} d \tau\right) d s \\
\geq & R_{3} \frac{\beta_{1}-2}{\Gamma\left(\beta_{1}\right)} K_{1}(t) \int_{\sigma}^{1-\sigma} L_{1}(s) \phi_{1}^{-1}\left(\int_{\sigma}^{1-\sigma} a_{1}(\tau) H_{1}\left(d_{1}, \tau\right) l_{1} d \tau\right) d s \\
\geq & R_{3} \frac{\beta_{1}-2}{\Gamma\left(\beta_{1}\right)} K_{1}(t) \int_{\sigma}^{1-\sigma} L_{1}(s) \phi_{1}^{-1}\left(\int_{\sigma}^{1-\sigma} a_{1}(\tau) H_{1}\left(d_{1}, \tau\right)\right. \\
& \left.\left.+\phi_{1}\left(\frac{\Gamma\left(\beta_{1}\right)}{2 c_{1}(1-2 \sigma)^{2}\left(\beta_{1}-2\right)}\right)\right) d \tau\right) d s \\
> & \left.R_{3} \frac{\beta_{1}-2}{\Gamma\left(\beta_{1}\right)} K_{1}(t) \int_{\sigma}^{1-\sigma} L_{1}(s) \phi_{1}^{-1}\left(\int_{\sigma}^{1-\sigma} \phi_{1}\left(\frac{\Gamma\left(\beta_{1}\right)}{2 c_{1}(1-2 \sigma)^{2}\left(\beta_{1}-2\right)}\right)\right) d \tau\right) d s \\
> & \frac{R_{3}}{2} .
\end{aligned}
$$

Similarly, we have

$$
\begin{aligned}
y_{1}(t) \geq & B\left(x_{1}(t), y_{1}(t)\right) \\
\geq & \frac{\beta_{2}-2}{\Gamma\left(\beta_{2}\right)} K_{2}(t) \int_{\sigma}^{1-\sigma} L_{2}(s) \phi_{2}^{-1}\left(\int_{\sigma}^{1-\sigma} a_{2}(\tau) H_{2}(s, \tau) \phi_{2}\left(x_{0}(\tau)+y_{0}(\tau)\right) l_{2} d \tau\right) d s . \\
\geq & R_{3} \frac{\beta_{2}-2}{\Gamma\left(\beta_{2}\right)} K_{2}(t) \int_{\sigma}^{1-\sigma} L_{2}(s) \phi_{2}^{-1}\left(\int_{\sigma}^{1-\sigma} a_{2}(\tau) H_{2}\left(d_{2}, \tau\right) l_{2} d \tau\right) d s . \\
\geq & R_{3} \frac{\beta_{1}-2}{\Gamma\left(\beta_{2}\right)} K_{2}(t) \int_{\sigma}^{1-\sigma} L_{2}(s) \phi_{2}^{-1}\left(\int_{\sigma}^{1-\sigma} a_{2}(\tau) H_{2}\left(d_{2}, \tau\right)\right. \\
& \left.+\phi_{2}\left(\frac{\Gamma\left(\beta_{2}\right)}{2 c_{2}(1-2 \sigma)^{2}\left(\beta_{2}-2\right)}\right) d \tau\right) d s \\
> & \left.R_{3} \frac{\beta_{2}-2}{\Gamma\left(\beta_{2}\right)} K_{2}(t) \int_{\sigma}^{1-\sigma} L_{2}(s) \phi_{2}^{-1}\left(\int_{\sigma}^{1-\sigma} \phi_{2}\left(\frac{\Gamma\left(\beta_{2}\right)}{2 c_{2}(1-2 \sigma)^{2}\left(\beta_{2}-2\right)}\right)\right) d \tau\right) d s \\
> & \frac{R_{3}}{2} .
\end{aligned}
$$

Consequently

$$
\left\|\left(x_{1}, y_{1}\right)\right\|>R_{3},
$$

contradicting $\left\|\left(x_{1}, y_{1}\right)\right\|=R_{3}$. Making use of Lemma 2.3, we conclude that

$$
i\left(T, \Omega_{3} \cap \mathcal{P}, \mathcal{P}\right)=0 \text {. and } i\left(T,\left(\Omega_{3} \backslash \overline{\Omega_{2}}\right) \cap \mathcal{P}, \mathcal{P}\right)=-1 \text {. }
$$

Finally, operator $T$ has a fixed point $\left(x_{1}^{*}, y_{1}^{*}\right)$ such that $R_{2}<\left\|\left(x_{1}^{*}, y_{1}^{*}\right)\right\|<$ $R_{3}$. Since $R_{1}<\left\|\left(x_{0}, y_{0}\right)\right\|<R_{2}<\left\|\left(x_{1}^{*}, y_{1}^{*}\right)\right\|<R_{3}$, then $T$ has two positive fixed points.

Example 3.1. Consider the bvp:

$$
\left\{\begin{array}{l}
-D_{0^{+}}^{\frac{3}{2}}\left(\left(D_{0^{+}}^{\frac{7}{2}} x(t)\right)^{p}\right)=\frac{12 \delta^{p}}{10} \frac{6^{p}\left(1-2^{-p}\right) \sqrt{\pi}}{5^{p}}(1-t)^{\frac{3}{2}}(t+1)\left(x^{q}+y^{q}+1\right)^{\frac{1}{p}} \\
-D_{0^{+}}^{\frac{7}{4}}\left(\left(D_{0^{+}}^{\frac{15}{4}} y(t)\right)^{p}\right)=\left(\frac{64 \delta}{49}\right)^{p} \frac{3}{4}\left(1-2^{-2 p}\right) \sqrt{\pi}(1-t)^{\frac{1}{4}}(t+1)\left((x+y)^{q}+1\right)^{\frac{1}{p}} \\
x(0)=x(1)=x^{\prime}(0)=x^{\prime}(1)=0, D_{0^{+}}^{\frac{7}{2}} x(0)=0, D_{0^{+}}^{\frac{7}{2}} x(1)=2^{\frac{1}{2 p}-1} D_{0^{+}}^{\frac{7}{2}} x\left(\frac{1}{2}\right) \\
y(0)=y(1)=y^{\prime}(0)=y^{\prime}(1)=0, D_{0^{+}}^{\frac{15}{4}} y(0)=0, D_{0^{+}}^{\frac{15}{4}} y(1)=2^{\frac{3}{2 p}-2} D_{0^{+}}^{\frac{15}{4}} y\left(\frac{1}{4}\right),
\end{array}\right.
$$


where

$$
\begin{aligned}
& f(t, x, y)=\frac{6^{p}\left(1-2^{-p}\right) \sqrt{\pi}}{5^{p}}(t+1)\left(x^{q}+y^{q}+1\right)^{\frac{1}{p}} \\
& g(t, x, y)=\frac{3}{4}\left(1-2^{-2 p}\right) \sqrt{\pi}(t+1)\left((x+y)^{q}+1\right)^{\frac{1}{p}},
\end{aligned}
$$

and

$$
a_{1}(t)=\frac{12 \delta^{p}}{10}(1-t)^{\frac{3}{2}}, a_{2}(t)=\left(\frac{64 \delta}{49}\right)^{p}(1-t)^{\frac{1}{4}}(\delta>0) .
$$

$p$ is an odd number, $q>p^{2}$, and $\phi_{1}(t)=\phi_{2}(t)=t^{p}$ is the $p$-Laplacian. As a consequence, for $i \in\{1,2\}, \phi_{i}$ are continuous, increasing, homeomorphism, multiplicative and $\phi_{i}(0)=0$.

Let

$$
\psi_{1}(x, y)=\left(x^{q}+y^{q}+1\right)^{\frac{1}{p}}, \psi_{2}(x, y)=\left((x+y)^{q}+1\right)^{\frac{1}{p}}
$$

and

$$
m_{1}(t)=\frac{6^{p}\left(1-2^{-p}\right) \sqrt{\pi}}{5^{p}}(t+1), m_{2}(t)=\frac{3}{4}\left(1-2^{-2 p}\right) \sqrt{\pi}(t+1) .
$$

Thus Hypothesis $\left(\mathcal{H}_{1}\right)$ is easily checked. Moreover, we can verify the rest of hypotheses:

$\left(\mathcal{H}_{\mathbf{2}}\right)$

$$
\begin{aligned}
& \sup _{c>0} \frac{c}{\sum_{i=1}^{2} \frac{M_{i}}{\Gamma\left(\beta_{i}\right)} \phi^{-1}\left(\lambda_{i} \psi_{i}(c, c) \int_{0}^{1}(1-\tau)^{\alpha_{i}-1} a_{i}(\tau) m_{i}(\tau) d \tau\right)} \\
& >\sup _{c>0} \frac{c}{\delta\left(\left(2 c^{q}+1\right)^{\frac{1}{p^{2}}}+2\left(2^{q} c^{q}+1\right)^{\frac{1}{p^{2}}}\right)} .
\end{aligned}
$$

$\left(\mathcal{H}_{3}\right)$

$$
\begin{gathered}
\min _{t \in[\sigma, 1-\sigma]} a_{1}(t)=\min _{t \in[\sigma, 1-\sigma]} \frac{12 \delta^{p}}{10}(1-t)^{\frac{3}{2}}=\frac{12 \delta^{p}}{10} \sigma^{\frac{3}{2}} \neq 0, \\
\min _{t \in[\sigma, 1-\sigma]} a_{2}(t) \\
=\min _{t \in[\sigma, 1-\sigma]}\left(\frac{64 \delta}{49}\right)^{p}(1-t)^{\frac{1}{4}}=\left(\frac{64 \delta}{49}\right)^{p} \sigma^{\frac{1}{4}} \neq 0,
\end{gathered}
$$

and

$$
\begin{gathered}
\lim _{x+y \rightarrow+\infty} \inf _{t \in[\sigma, 1-\sigma]} \frac{f(t, x, y)}{\phi_{1}(x+y)} \\
=\lim _{x+y \rightarrow+\infty} \inf _{t \in[\sigma, 1-\sigma]} \frac{\frac{6^{p}\left(1-2^{-p}\right) \sqrt{\pi}}{5^{p}}(t+1)\left(x^{q}+y^{q}+1\right)^{\frac{1}{p}}}{(x+y)^{p}}=+\infty,
\end{gathered}
$$




$$
\begin{gathered}
\lim _{x+y \rightarrow \infty} \inf _{t \in[\sigma, 1-\sigma]} \frac{g(t, x, y)}{\phi_{2}(x+y)} \\
=\lim _{x+y \rightarrow \infty} \inf _{t \in[\sigma, 1-\sigma]} \frac{\frac{3}{4}\left(1-2^{-2 p}\right) \sqrt{\pi}(t+1)\left((x+y)^{q}+1\right)^{\frac{1}{p}}}{(x+y)^{p}}=+\infty .
\end{gathered}
$$

$\left(\mathcal{H}_{4}\right)$

$$
\begin{gathered}
\lim _{x+y \rightarrow 0} \inf _{t \in[\sigma, 1-\sigma]} \frac{f(t, x, y)}{\phi_{1}(x+y)} \\
=\lim _{x+y \rightarrow 0} \inf _{t \in[\sigma, 1-\sigma]} \frac{\frac{6^{p}\left(1-2^{-p}\right) \sqrt{\pi}}{5^{p}}(t+1)\left(x^{q}+y^{q}+1\right)^{\frac{1}{p}}}{(x+y)^{p}}=+\infty,
\end{gathered}
$$

and

$$
\begin{gathered}
\lim _{x+y \rightarrow 0} \inf _{t \in[\sigma, 1-\sigma]} \frac{g(t, x, y)}{\phi_{2}(x+y)} \\
\lim _{x+y \rightarrow 0} \inf _{t \in[\sigma, 1-\sigma]} \frac{\frac{3}{4}\left(1-2^{-2 p}\right) \sqrt{\pi}(t+1)\left((x+y)^{q}+1\right)^{\frac{1}{p}}}{(x+y)^{p}}=+\infty .
\end{gathered}
$$

Finally we can choose $\delta$ such that $0<\delta<\sup _{c>0} \frac{c}{\left(\left(2 c^{q}+1\right)^{\frac{1}{p^{2}}}+2\left(2^{q} c^{q}+1\right)^{\frac{1}{p^{2}}}\right)}$. Then the hypotheses of Theorem 3.1 are satisfied and problem (3.5) has at least two positive solutions.

\section{Nonlinearities with one singularity}

In this section, we suppose that the nonlinear functions $f, g:[0,1] \times \mathbf{R}_{*}^{+} \times$ $\mathbf{R}^{+} \rightarrow \mathbf{R}^{+}$are continuous and satisfy

$\left(\mathcal{A}_{\mathbf{1}}\right)$ For $i \in\{0,1\}$, there exist $m_{i} \in C\left([0,1], \mathbf{R}^{+}\right), \psi_{i} \in C\left(\mathbf{R}^{+} \times \mathbf{R}^{+}, \mathbf{R}^{+}\right)$ increasing functions in each argument, and $q_{i}, p_{i}, \in C\left(\mathbf{R}_{*}^{+}, \mathbf{R}^{+}\right)$such that $p_{i}$ are decreasing functions and $\frac{q_{i}}{p_{i}}$ are increasing functions with

$$
\begin{aligned}
& f(t, x, y) \leq m_{1}(t) q_{1}(x) \psi_{1}(x, y), \quad \forall(t, x, y) \in[0,1] \times \mathbf{R}_{*}^{+} \times \mathbf{R}^{+} \\
& g(t, x, y) \leq m_{2}(t) q_{2}(x) \psi_{2}(x, y), \quad \forall(t, x, y) \in[0,1] \times \mathbf{R}_{*}^{+} \times \mathbf{R}^{+}
\end{aligned}
$$

and for each $c>0$,

$$
\int_{0}^{1}(1-\tau)^{\alpha_{1}-1} a_{i}(\tau) m_{i}(\tau) p_{i}(c \gamma(\tau)) d \tau<+\infty
$$


$\left(\mathcal{A}_{\mathbf{2}}\right)$ For all $0<\omega<1$,

$\sup _{c>0} \frac{c}{\sum_{i=1}^{2} \frac{M_{i}}{\Gamma\left(\beta_{i}\right)} \phi_{i}^{-1}\left(\lambda_{i} \frac{q_{i}(c)}{p_{i}(c)} \psi_{i}(c, c) \int_{0}^{1}(1-\tau)^{\alpha_{i}-1} a_{i}(\tau) m_{i}(\tau) p_{i}(\omega \gamma(\tau)) d \tau\right)}>1$.

$\left(\mathcal{A}_{3}\right)$ There exists $\sigma \in\left(0, \frac{1}{2}\right)$ such that

$$
\lim _{x+y \rightarrow+\infty} \inf _{t \in[\sigma, 1-\sigma]} \frac{f(t, x, y)}{\phi_{1}(x+y)} \geq l_{1}
$$

and

$$
\lim _{x+y \rightarrow+\infty} \inf _{t \in[\sigma, 1-\sigma]} \frac{g(t, x, y)}{\phi_{2}(x+y)} \geq l_{2}
$$

where

$$
l_{i}=1+\frac{\phi_{i}\left(\frac{\Gamma\left(\beta_{i}\right)}{2 c_{i}(1-2 \sigma)^{2}\left(\beta_{i}-2\right)}\right)}{\int_{\sigma}^{1-\sigma} a_{i}(\tau) H_{i}\left(d_{i}, \tau\right) d \tau}, \quad i \in\{1,2\},
$$

$\mathrm{c}_{i}=\min _{(t, s) \in([\sigma, 1-\sigma])^{2}} K_{i}(t) L_{i}(s), \min _{t \in[\sigma, 1-\sigma]} a_{i}(\tau) \neq 0 \operatorname{and} H_{i}\left(d_{i}, \tau\right)=\min _{s \in[\sigma, 1-\sigma]} H_{i}(s, \tau)$.

$\left(\mathcal{A}_{4}\right)$ For every $c>0$ and $\frac{1}{4}<\theta<\frac{1}{2}$, there exist $\rho_{c}, \rho_{c}^{*} \in C\left([0,1], \mathbf{R}^{+}\right)$such that $\rho_{c}(t)>0, \rho_{c}^{*}(t)>0$ on $\left(\theta-\frac{1}{4}, \theta\right)$ and

$$
\begin{aligned}
& f(t, x, y) \geq \rho_{c}(t), \quad \forall t \in[0,1], \forall x, y \in(0, c], \\
& g(t, x, y) \geq \rho_{c}^{*}(t), \quad \forall t \in[0,1], \forall x, y \in(0, c] .
\end{aligned}
$$
by

Define two sequences of approximating functions $\left\{f_{n}\right\}_{n \geq 1}$ and $\left\{g_{n}\right\}_{n \geq 1}$

$$
\begin{array}{ll}
f_{n}(t, x, y)=f\left(t, \max \left\{\frac{1}{n}, x\right\}, y\right), & n \in\{1,2, \ldots\} \\
g_{n}(t, x, y)=g\left(t, \max \left\{\frac{1}{n}, x\right\}, y\right), & n \in\{1,2, \ldots\}
\end{array}
$$

for $(t, x, y) \in(0,1) \times \mathcal{P}$ and define the sequences of operators:

$$
\begin{gathered}
A_{n}(x, y)(t)=\int_{0}^{1} G_{1}(t, s) \phi_{1}^{-1}\left(\int_{0}^{1} H_{1}(s, \tau) a_{1}(\tau) f_{n}(\tau, x(\tau, y(\tau)) d \tau) d s\right. \\
B_{n}(x, y)(t)=\int_{0}^{1} G_{2}(t, s) \phi_{2}^{-1}\left(\int_{0}^{1} H_{2}(s, \tau) a_{2}(\tau) g_{n}(\tau, x(\tau, y(\tau)) d \tau) d s\right. \\
T_{n}(x, y)=\left(A_{n}(x, y), B_{n}(x, y)\right) .
\end{gathered}
$$

We have,

Proposition 4.1. Assume that condition $\left(\mathcal{A}_{1}\right)$ holds. Then for each $n \geq 1$, the operator $T_{n}: P \longrightarrow P$ is completely continuous. 
Proof. Step 1: We claim that for $(x, y) \in \mathcal{P}$ we have $T_{n}(x, y) \in \mathcal{P}$. We have $A_{n}(x, y)(t) \geq 0$ and $B_{n}(x, y)(t) \geq 0 ; \forall t \in[0,1]$. By $(c)$ of Lemma 2.6, we have

$$
\begin{aligned}
A_{n}(x, y)(t) & =\int_{0}^{1} G_{1}(t, s) \phi_{1}^{-1}\left(\int_{0}^{1} H_{1}(s, \tau) a_{1}(\tau) f_{n}(\tau, x(\tau), y(\tau)) d \tau\right) d s \\
& \geq \frac{\beta_{1}-2}{M_{1}} \gamma(t) \int_{0}^{1} \sup _{t \in[0,1]} G_{1}(t, s) \phi_{1}^{-1}\left(\int_{0}^{1} H_{1}(s, \tau) a_{1}(\tau) f_{n}(\tau, x(\tau), y(\tau)) d \tau\right) d s \\
& \geq \frac{\beta_{1}-2}{M_{1}} \gamma(t) \sup _{t \in[0,1]} \int_{0}^{1} G_{1}(t, s) \phi_{1}^{-1}\left(\int_{0}^{1} H_{1}(s, \tau) a_{1}(\tau) f_{n}(\tau, x(\tau), y(\tau)) d \tau\right) d s \\
& \geq \frac{\beta_{1}-2}{M_{1}} \gamma(t)\left\|A_{n}(x, y)\right\| .
\end{aligned}
$$

Similarly we have by $(c)$ of Lemma 2.6

$$
\begin{aligned}
B_{n}(x, y)(t) & =\int_{0}^{1} G_{2}(t, s) \phi_{2}^{-1}\left(\int_{0}^{1} H_{2}(s, \tau) a_{2}(\tau) g_{n}(\tau, x(\tau), y(\tau)) d \tau\right) d s \\
& \geq \frac{\beta_{2}-2}{M_{2}} \gamma(t) \int_{0}^{1} \sup _{t \in[0,1]} G_{2}(t, s) \phi_{2}^{-1}\left(\int_{0}^{1} H_{2}(s, \tau) a_{2}(\tau) g_{n}(\tau, x(\tau), y(\tau)) d \tau\right) d s \\
& \geq \frac{\beta_{2}-2}{M_{2}} \gamma(t) \sup _{t \in[0,1]} \int_{0}^{1} G_{2}(t, s) \phi_{2}^{-1}\left(\int_{0}^{1} H_{2}(s, \tau) a_{2}(\tau) g_{n}(\tau, x(\tau), y(\tau)) d \tau\right) d s \\
& \geq \frac{\beta_{2}-2}{M_{2}} \gamma(t)\left\|B_{n}(x, y)\right\| .
\end{aligned}
$$

Hence $T_{n}(\mathcal{P}) \subset \mathcal{P}$.

Step 2: Let $D$ is a bounded set, then there exists $r>1$ such that

$$
\forall(x, y) \in D,\|(x, y)\| \leq r .
$$

Hypothesis $\left(\mathcal{A}_{1}\right)$ and the properties $(b)$ and $(d)$ of Lemma 2.6 lead to the following estimates:

$$
\begin{aligned}
\left\|A_{n}(x, y)\right\|= & \left\|\int_{0}^{1} G_{1}(t, s) \phi_{1}^{-1}\left(\int_{0}^{1} H_{1}(s, \tau) a_{1}(\tau) f_{n}(\tau, x(\tau), y(\tau)) d \tau\right) d s\right\| \\
\leq & \frac{M_{1}}{\Gamma\left(\beta_{1}\right)} \phi_{1}^{-1}\left(\int_{0}^{1} a_{1}(\tau) \lambda_{1}(1-\tau)^{\alpha_{1}-1} f\left(\tau, \max \left\{\frac{1}{n}, x(\tau)\right\}, y(\tau)\right) d \tau\right) \\
\leq & \frac{M_{1}}{\Gamma\left(\beta_{1}\right)} \phi_{1}^{-1}\left(\lambda_{1} \int_{0}^{1} a_{1}(\tau)(1-\tau)^{\alpha_{1}-1} m_{1}(\tau) q_{1}\left(\max \left\{\frac{1}{n}, x(\tau)\right\}\right)\right. \\
& \left.\psi_{1}\left(\max \left\{\frac{1}{n}, x(\tau)\right\}, y\right)(\tau) d \tau\right) \\
\leq & \frac{M_{1}}{\Gamma\left(\beta_{1}\right)} \phi_{1}^{-1}\left(\lambda_{1} \int_{0}^{1} a_{1}(\tau)(1-\tau)^{\alpha_{1}-1} m_{1}(\tau) p_{1}\left(\max \left\{\frac{1}{n}, x(\tau)\right\}\right)\right. \\
& \frac{q_{1}\left(\max \left\{\frac{1}{n}, x(\tau)\right\}\right)}{p_{1}\left(\max \left\{\frac{1}{n}, x(\tau)\right\}\right)} \psi_{1}\left(\max \left\{\frac{1}{n}, x(\tau)\right\}, y(\tau)\right) d \tau \\
\leq & \frac{M_{1}}{\Gamma\left(\beta_{1}\right)} \phi_{1}^{-1}\left(\lambda_{1} \frac{q_{1}(r)}{p_{1}(r)} \psi_{1}(r, r) \int_{0}^{1} a_{1}(\tau)(1-\tau)^{\alpha_{1}-1} m_{1}(\tau) p_{1}\left(\frac{1}{n}\right) d \tau\right) \\
\leq & \frac{M_{1}}{\Gamma\left(\beta_{1}\right)} \phi_{1}^{-1}\left(\lambda_{1} \frac{q_{1}(r)}{p_{1}(r)} \psi_{1}(r, r) \int_{0}^{1} a_{1}(\tau) m_{1}(\tau)(1-\tau)^{\alpha_{1}-1} p_{1}\left(\frac{1}{n} \gamma(\tau)\right) d \tau\right) \\
< & +\infty
\end{aligned}
$$


Similarly,

$$
\begin{aligned}
\left\|B_{n}(x, y)\right\| & \leq \frac{M_{2}}{\Gamma\left(\beta_{2}\right)} \phi_{2}^{-1}\left(\lambda_{2} \frac{q_{2}(r)}{p_{2}(r)} \psi_{2}(r, r) \int_{0}^{1} a_{2}(\tau) m_{2}(\tau)(1-\tau)^{\alpha_{2}-1} p_{2}\left(\frac{1}{n} \gamma(\tau)\right) d \tau\right) \\
& <+\infty
\end{aligned}
$$

Step 3: For $t, t^{\prime} \in[0,1]\left(t<t^{\prime}\right)$, we have

$$
\begin{aligned}
& \left|A_{n}(x, y)(t)-A_{n}(x, y)\left(t^{\prime}\right)\right| \\
\leq & \int_{0}^{1}\left|G_{1}(t, s)-G_{1}\left(t^{\prime}, s\right)\right| \phi_{1}^{-1}\left(\int_{0}^{1} a_{1}(\tau) H_{1}(s, \tau) f_{n}(\tau, x(\tau), y(\tau)) d \tau\right) d s .
\end{aligned}
$$

In the same way

$$
\begin{aligned}
& \left|B_{n}(x, y)(t)-B_{n}(x, y)\left(t^{\prime}\right)\right| \\
\leq & \int_{0}^{1}\left|G_{2}(t, s)-G_{2}\left(t^{\prime}, s\right)\right| \phi_{2}^{-1}\left(\int_{0}^{1} a_{2}(\tau) H_{2}(s, \tau) g_{n}(\tau, x(\tau), y(\tau)) d \tau\right) d s .
\end{aligned}
$$

By the continuity of $G_{1}, G_{2}$ and Arzela-Ascoli Lemma, $T_{n}$ is a completely continuous.

Theorem 4.1. Assume that Assumptions $\left(\mathcal{A}_{1}\right)-\left(\mathcal{A}_{4}\right)$ hold. Then problem (1.1) has at least two positive solutions.

Proof. Step 1: An approximating solution. From condition $\left(\mathcal{A}_{2}\right)$, there exists $R_{1}>0$ such that, for all $0<\omega<1$

$\frac{R_{1}}{\sum_{i=1}^{2} \frac{M_{i}}{\Gamma\left(\beta_{i}\right)} \phi^{-1}\left(\lambda_{i} \frac{q_{i}\left(R_{1}\right)}{p_{i}\left(R_{1}\right)} \psi_{i}\left(R_{1}, R_{1}\right) \int_{0}^{1} a_{i}(\tau) m_{i}(\tau)(1-\tau)^{\alpha_{i}-1} p_{i}(\omega \gamma(\tau)) d \tau\right)}>1$.

Let

$$
\Omega_{1}=\left\{(x, y) \in E:\|(x, y)\|<R_{1}\right\}
$$

We claim that $(x, y) \neq \lambda T_{n}(x, y)$ for all $x \in \partial \Omega_{1} \cap \mathcal{P}, \lambda \in(0,1]$ and $n \geq$ $n_{0} \geq \frac{1}{R_{1}}$. If not, suppose that there exist $n_{1} \geq n_{0},\left(x_{1}, y_{1}\right) \in \partial \Omega_{1} \cap \mathcal{P}$ and $\lambda_{1} \in(0,1]$ such that $\left(x_{1}, y_{1}\right)=\lambda_{1} T_{n_{1}}\left(x_{1}, y_{1}\right)$. As a consequence, we obtain 
the estimates:

$$
\begin{aligned}
\left\|x_{1}\right\| \leq & \left\|\lambda_{1} A_{n_{1}}\left(x_{1}, y_{1}\right)\right\| \\
\leq & \left\|A_{n_{1}}\left(x_{1}, y_{1}\right)\right\| \\
\leq & \left\|\int_{0}^{1} G_{1}(t, s) \phi_{1}^{-1}\left(\int_{0}^{1} a_{1}(\tau) H_{1}(s, \tau) f_{n_{1}}\left(\tau, x_{1}(\tau), y_{1}(\tau)\right) d \tau\right) d s\right\| \\
\leq & \frac{M_{1}}{\Gamma\left(\beta_{1}\right)} \phi_{1}^{-1}\left(\int_{0}^{1} a_{1}(\tau) \lambda_{1}(1-\tau)^{\alpha_{1}-1} f\left(\tau, \max \left\{\frac{1}{n_{1}}, x_{1}(\tau)\right\}, y_{1}(\tau)\right) d \tau\right) \\
\leq & \frac{M_{1}}{\Gamma\left(\beta_{1}\right)} \phi_{1}^{-1}\left(\lambda_{1} \int_{0}^{1} a_{1}(\tau)(1-\tau)^{\alpha_{1}-1} m_{1}(\tau) q_{1}\left(\max \left\{\frac{1}{n_{1}}, x_{1}(\tau)\right\}\right)\right. \\
& \left.\psi_{1}\left(\max \left\{\frac{1}{n_{1}}, x_{1}(\tau)\right\}, y_{1}\right)(\tau) d \tau\right) \\
\leq & \frac{M_{1}}{\Gamma\left(\beta_{1}\right)} \phi_{1}^{-1}\left(\lambda_{1} \int_{0}^{1} a_{1}(\tau)(1-\tau)^{\alpha_{1}-1} m_{1}(\tau) p_{1}\left(\max \left\{\frac{1}{n_{1}}, x_{1}(\tau)\right\}\right)\right. \\
& \frac{q_{1}\left(\max \left\{\frac{1}{n_{1}}, x_{1}(\tau)\right\}\right)}{p_{1}\left(\max \left\{\frac{1}{n_{1}}, x_{1}(\tau)\right\}\right)} \psi_{1}\left(\max \left\{\frac{1}{n_{1}}, x_{1}(\tau)\right\}, y_{1}(\tau)\right) d \tau \\
\leq & \frac{M_{1}}{\Gamma\left(\beta_{1}\right)} \phi_{1}^{-1}\left(\lambda_{1} \frac{q_{1}\left(R_{1}\right)}{p_{1}\left(R_{1}\right)} \psi_{1}\left(R_{1}, R_{1}\right) \int_{0}^{1} a_{1}(\tau)(1-\tau)^{\alpha_{1}-1} m_{1}(\tau) p_{1}\left(\frac{1}{n_{1}}\right) d \tau\right) \\
\leq & \frac{M_{1}}{\Gamma\left(\beta_{1}\right)} \phi_{1}^{-1}\left(\lambda_{1} \frac{q_{1}\left(R_{1}\right)}{p_{1}\left(R_{1}\right)} \psi_{1}\left(R_{1}, R_{1}\right) \int_{0}^{1} a_{1}(\tau) m_{1}(\tau)(1-\tau)^{\alpha_{1}-1} p_{1}\left(\frac{1}{n_{1}} \gamma(\tau)\right) d \tau\right) .
\end{aligned}
$$

In a similar way

$$
\begin{aligned}
\left\|y_{1}\right\| & =\left\|\lambda_{1} B_{n_{1}}\left(x_{1}, y_{1}\right)\right\| \\
& \leq\left\|B_{n_{1}}\left(x_{1}, y_{1}\right)\right\| \\
& \leq\left\|\int_{0}^{1} G_{2}(t, s) \phi_{2}^{-1}\left(\int_{0}^{1} a_{2}(\tau) H_{2}(s, \tau) g_{n_{1}}\left(\tau, x_{1}(\tau), y_{1}(\tau)\right) d \tau\right) d s\right\|, \\
& \leq \frac{M_{2}}{\Gamma\left(\beta_{2}\right)} \phi_{2}^{-1}\left(\lambda_{2} \frac{q_{2}\left(R_{1}\right)}{p_{2}\left(R_{1}\right)} \psi_{2}\left(R_{1}, R_{1}\right) \int_{0}^{1} a_{2}(\tau)(1-\tau)^{\alpha_{2}-1} m_{2}(\tau) p_{2}\left(\frac{1}{n_{1}}\right) d \tau\right) \\
& \leq \frac{M_{2}}{\Gamma\left(\beta_{2}\right)} \phi_{2}^{-1}\left(\lambda_{2} \frac{q_{2}\left(R_{1}\right)}{p_{2}\left(R_{1}\right)} \psi_{2}\left(R_{1}, R_{1}\right) \int_{0}^{1} a_{2}(\tau) m_{2}(\tau)(1-\tau)^{\alpha_{2}-1} p_{2}\left(\frac{1}{n_{1}} \gamma(\tau)\right) d \tau\right) .
\end{aligned}
$$

Then,

$$
\begin{aligned}
R_{1} & =\left\|\left(x_{1}, y_{1}\right)\right\| \\
& \leq \frac{M_{1}}{\Gamma\left(\beta_{1}\right)} \phi_{1}^{-1}\left(\lambda_{1} \frac{q_{1}\left(R_{1}\right)}{p_{1}\left(R_{1}\right)} \psi_{1}\left(R_{1}, R_{1}\right) \int_{0}^{1} a_{1}(\tau) m_{1}(\tau)(1-\tau)^{\alpha_{1}-1} p_{1}\left(\frac{1}{n_{1}} \gamma(\tau)\right) d \tau\right) \\
& +\frac{M_{2}}{\Gamma\left(\beta_{2}\right)} \phi_{2}^{-1}\left(\lambda_{2} \frac{q_{2}\left(R_{1}\right)}{p_{2}\left(R_{1}\right)} \psi_{2}\left(R_{1}, R_{1}\right) \int_{\sigma}^{1-\sigma} a_{2}(\tau) m_{2}(\tau)(1-\tau)^{\alpha_{2}-1} p_{2}\left(\frac{1}{n_{1}} \gamma(\tau)\right) d \tau\right)
\end{aligned}
$$

which is a contradiction to (4.3). By Lemma 2.2, we infer that

$$
i\left(T_{n}, \Omega_{1} \cap \mathcal{P}, \mathcal{P}\right)=1, \text { for all } n \in\left\{n_{0}, n_{0}+1, \ldots\right\} .
$$

Hence there exists $\left(x_{n}, y_{n}\right) \in \Omega_{1} \cap \mathcal{P}$ such that $T_{n}\left(x_{n}, y_{n}\right)=\left(x_{n}, y_{n}\right)$ and $\left\|\left(x_{n}, y_{n}\right)\right\|<R_{1}$, for all $n \geq n_{0}$.

\section{Step 2: a compactness argument.}

(a) Since $\left\|\left(x_{n}, y_{n}\right)\right\|<R_{1}$, by $\left(\mathcal{A}_{4}\right)$ there exist functions $\rho_{R_{1}}, \rho_{R_{1}}^{*} \in$ $C\left([0,1], \mathbf{R}^{+}\right)$such that

$$
f_{n}\left(t, x_{n}(t), y_{n}(t)\right) \geq \rho_{R_{1}}(t), \quad \forall t \in[0,1]
$$


and

$$
g_{n}\left(t, x_{n}(t), y_{n}(t)\right) \geq \rho_{R_{1}}^{*}(t), \quad \forall t \in[0,1]
$$

Then

$$
\begin{aligned}
& x_{n}(t)=A_{n}\left(x_{n}, y_{n}\right)(t) \\
& =\int_{0}^{1} G_{1}(t, s) \phi_{1}^{-1}\left(\int_{0}^{1} a_{1}(\tau) H_{1}(s, \tau) f_{n}\left(\tau, x_{n}(\tau), y_{n}(\tau)\right) d \tau\right) d s \\
& \geq \int_{\theta}^{1-\theta} \frac{\left(\beta_{1}-2\right) K_{1}(t) L_{1}(s)}{\Gamma\left(\beta_{1}\right)} \phi_{1}^{-1}\left(\int_{\theta-\frac{1}{4}}^{\theta} a_{1}(\tau) H_{1}(s, \tau) \rho_{R_{1}}(\tau) d \tau\right) d s \\
& \geq \frac{\phi_{1}^{-1}\left(\frac{1}{\Gamma\left(\alpha_{1}\right)}\right)\left(\beta_{1}-2\right) K_{1}(t)}{\Gamma\left(\beta_{1}\right)} \\
& \int_{\theta}^{1-\theta} L_{1}(s) \phi_{1}^{-1}\left(\int_{\theta-\frac{1}{4}}^{\theta} a_{1}(\tau)\left[[s(1-\tau)]^{\alpha_{1}-1}-(s-\tau)^{\alpha_{1}-1}\right] \rho_{R_{1}}(\tau) d \tau\right) d s
\end{aligned}
$$

and

$$
\begin{aligned}
y_{n}(t)= & B_{n}\left(x_{n}, y_{n}\right)(t) \\
= & \int_{0}^{1} G_{2}(s, \tau) \phi_{2}^{-1}\left(\int_{0}^{1} a_{2}(\tau) H_{2}(s, \tau) g_{n}\left(\tau, x_{n}(\tau), y_{n}(\tau)\right) d \tau\right) d s \\
\geq & \int_{\theta}^{1-\theta} \frac{\left(\beta_{2}-2\right) K_{2}(t) L_{2}(s)}{\Gamma\left(\beta_{2}\right)} \phi_{2}^{-1}\left(\int_{\theta-\frac{1}{4}}^{\theta} a_{2}(\tau) H_{2}(s, \tau) g_{n}\left(\tau, x_{n}(\tau), y_{n}(\tau)\right) d \tau\right) d s \\
\geq & \frac{\phi_{2}^{-1}\left(\frac{1}{\Gamma\left(\alpha_{2}\right)}\right)\left(\beta_{2}-2\right) K_{2}(t)}{\Gamma\left(\beta_{2}\right)} \\
& \int_{\theta}^{1-\theta} L_{2}(s) \phi_{2}^{-1}\left(\int_{\theta-\frac{1}{4}}^{\theta} a_{2}(\tau)\left[[s(1-\tau)]^{\alpha_{2}-1}-(s-\tau)^{\alpha_{2}-1}\right] \rho_{R_{1}}^{*}(\tau) d \tau\right) d s .
\end{aligned}
$$

Let the positive numbers

$$
\begin{gathered}
c_{1}^{*}=\frac{\phi_{1}^{-1}\left(\frac{1}{\Gamma\left(\alpha_{1}\right)}\right)\left(\beta_{1}-2\right)}{\Gamma\left(\beta_{1}\right)} \int_{\theta}^{1-\theta} L_{1}(s) \phi_{1}^{-1} \\
\left(\int_{\theta-\frac{1}{4}}^{\theta} a_{1}(\tau)\left[[s(1-\tau)]^{\alpha_{1}-1}-(s-\tau)^{\alpha_{1}-1}\right] \rho_{R_{1}}(\tau) d \tau\right) d s
\end{gathered}
$$

and

$$
\begin{gathered}
c_{2}^{*}=\frac{\phi_{2}^{-1}\left(\frac{1}{\Gamma\left(\alpha_{2}\right)}\right)\left(\beta_{2}-2\right)}{\Gamma\left(\beta_{2}\right)} \int_{\theta}^{1-\theta} L_{2}(s) \phi_{2}^{-1} \\
\left(\int_{\theta-\frac{1}{4}}^{\theta} a_{2}(\tau)\left[[s(1-\tau)]^{\alpha_{2}-1}-(s-\tau)^{\alpha_{2}-1}\right] \rho_{R_{1}}^{*}(\tau) d \tau\right) d s .
\end{gathered}
$$

Then,

$x_{n}(t) \geq c_{1}^{*} K_{1}(t) \geq c_{1}^{*} \gamma(t)$ and $y_{n}(t) \geq c_{2}^{*} K_{2}(t) \geq c_{2}^{*} \gamma(t) \forall t \in[0,1], \forall n \geq n_{0}$. 
(b) For any $t, t^{\prime} \in[0,1]\left(t>t^{\prime}\right)$, we have the estimates

$$
\begin{aligned}
& \left|x_{n}(t)-x_{n}\left(t^{\prime}\right)\right| \\
\leq & \int_{0}^{1}\left|G_{1}(t, s)-G_{1}\left(t^{\prime}, s\right)\right| \phi_{1}^{-1}\left(\int_{0}^{1} a_{1}(\tau) H_{1}(s, \tau) f_{n}\left(\tau, x_{n}(\tau), y_{n}(\tau)\right) d \tau\right) d s \\
\leq & \int_{0}^{1}\left|G_{1}(t, s)-G_{1}\left(t^{\prime}, s\right)\right| \phi_{1}^{-1}\left(\int_{0}^{1} a_{1}(\tau) \lambda_{1}(1-\tau)^{\alpha_{1}-1} m_{1}(\tau) p_{1}\right. \\
& \left(c_{1}^{*} \gamma(\tau)\right) \frac{q_{1}\left(R_{1}\right)}{p_{1}\left(R_{1}\right)} \psi_{1}\left(R_{1}, R_{1}\right) d \tau \\
\leq & \int_{0}^{1}\left|G_{1}(t, s)-G_{1}\left(t^{\prime}, s\right)\right| \phi_{1}^{-1}\left(\lambda_{1} \frac{q_{1}\left(R_{1}\right)}{p_{1}\left(R_{1}\right)} \psi_{1}\left(R_{1}, R_{1}\right)\right) \\
& \phi_{1}^{-1}\left(\int_{0}^{1} a_{1}(\tau) m_{1}(\tau)(1-\tau)^{\alpha_{1}-1} p_{1}\left(c_{1}^{*} \gamma(\tau) d \tau\right) .\right.
\end{aligned}
$$

Also

$$
\begin{aligned}
& \left|y_{n}(t)-y_{n}\left(t^{\prime}\right)\right| \\
\leq & \int_{0}^{1}\left|G_{2}(t, s)-G_{2}\left(t^{\prime}, s\right)\right| \phi_{2}^{-1}\left(\int_{0}^{1} a_{2}(\tau) H_{2}(s, \tau) g_{n}\left(\tau, x_{n}(\tau), y_{n}(\tau)\right) d \tau\right) d s \\
\leq & \int_{0}^{1}\left|G_{2}(t, s)-G_{2}\left(t^{\prime}, s\right)\right| \phi_{2}^{-1}\left(\lambda_{2} \frac{q_{2}\left(R_{1}\right)}{p_{2}\left(R_{1}\right)} \psi_{2}\left(R_{1}, R_{1}\right)\right) \\
& \phi_{2}^{-1}\left(\int_{0}^{1} a_{2}(\tau) m_{2}(\tau)(1-\tau)^{\alpha_{2}-1} p_{2}\left(c_{2}^{*} \gamma(\tau) d \tau\right) .\right.
\end{aligned}
$$

Therefore $\left\{\left(x_{n}, y_{n}\right)\right\}_{n \geq n_{0}}$ is relatively compact in $E$. By the continuity of $G_{1}, G_{2}$, there exists a subsequence $\left\{\left(x_{n_{k}}, y_{n_{k}}\right)\right\}_{k \geq 1}$ such that $\lim _{k \rightarrow+\infty}\left(x_{n_{k}}, y_{n_{k}}\right)=$ $\left(x_{0}, y_{0}\right)$. Hence $x_{0}(t) \geq c_{1}^{*} \gamma(t), y_{0}(t) \geq c_{2}^{*} \gamma(t), \quad \forall t \in[0,1]$. From (4.3), we have $\left\|\left(x_{0}, y_{0}\right)\right\|<R_{1}$. By the Lebesgue dominated convergence theorem and the continuity of $f$ and $g$, we deduce that

$$
\begin{aligned}
x_{0}(t) & =\lim _{k \rightarrow+\infty} x_{n_{k}}(t) \\
& =\lim _{k \rightarrow+\infty} \int_{0}^{1} G_{1}(t, s) \phi_{1}^{-1}\left(\int_{0}^{1} a_{1}(\tau) H_{1}(s, \tau) f_{n_{k}}\left(\tau, x_{n_{k}}(\tau), y_{n_{k}}(\tau)\right) d \tau\right) d s \\
& =\lim _{k \rightarrow+\infty} \int_{0}^{1} G_{1}(t, s) \phi_{1}^{-1}\left(\int_{0}^{1} a_{1}(\tau) H_{1}(s, \tau) f\left(s, \max \left\{\frac{1}{n_{k}}, x_{n_{k}}(s)\right\}, y_{n_{k}}(s)\right)\right. \\
& =\int_{0}^{1} G_{1}(t, s) \phi_{1}^{-1}\left(\int_{0}^{1} a_{1}(\tau) H_{1}(s, \tau) f\left(\tau, x_{0}(\tau), y_{0}(\tau)\right) d \tau\right) d s
\end{aligned}
$$

and

$$
\begin{aligned}
y_{0}(t) & =\lim _{k \rightarrow+\infty} x_{n_{k}}(t) \\
& =\lim _{k \rightarrow+\infty} \int_{0}^{1} G_{2}(t, s) \phi_{2}^{-1}\left(\int_{0}^{1} a_{2}(\tau) H_{2}(s, \tau) g_{n_{k}}\left(\tau, x_{n_{k}}(\tau), y_{n_{k}}(\tau)\right) d \tau\right) d s \\
& =\lim _{k \rightarrow+\infty} \int_{0}^{1} G_{2}(t, s) \phi_{2}^{-1}\left(\int_{0}^{1} a_{2}(\tau) H_{2}(s, \tau) g\left(s, \max \left\{\frac{1}{n_{k}}, x_{n_{k}}(s)\right\}, y_{n_{k}}(s)\right)\right. \\
& =\int_{0}^{1} G_{2}(t, s) \phi_{2}^{-1}\left(\int_{0}^{1} a_{2}(\tau) H_{2}(s, \tau) g\left(\tau, x_{0}(\tau), y_{0}(\tau)\right) d \tau\right) d s .
\end{aligned}
$$

Then $\left(x_{0}, y_{0}\right)$ is a positive solution of problem (1.1). Arguing as in the proof of Theorem 3.1 and using condition $\left(\mathcal{A}_{3}\right)$, we can choose a number $R_{2}$ and show that

$$
i\left(T_{n}, \Omega_{2} \cap \mathcal{P}, \mathcal{P}\right)=0,
$$


with

$$
\Omega_{2}=\left\{(x, y) \in \mathcal{P}:\|(x, y)\| \leq R_{2}\right\}
$$

and also

$$
i\left(T_{n},\left(\Omega_{2} \backslash \overline{\Omega_{1}}\right) \cap \mathcal{P}, \mathcal{P}\right)=-1, \text { for all } n \in\left\{n_{0}, n_{0}+1, \ldots\right\}
$$

Therefore $T_{n}$ has a second fixed point $\left(w_{n}, v_{n}\right) \in\left(\Omega_{2} \backslash \bar{\Omega}_{1}\right) \cap \mathcal{P}, \forall n \geq n_{0}$, such that $R_{1}<\left\|\left(w_{n}, v_{n}\right)\right\|<R_{2}, \forall n \geq n_{0}$. As above, we can show that $\left\{\left(w_{n}, v_{n}\right)\right\}_{n \geq n_{0}}$ has a subsequence $\left\{\left(w_{n_{j}}, v_{n_{j}}\right)\right\}_{j \geq 1}$ such that $\lim _{j \rightarrow+\infty}\left(w_{n_{j}}, v_{n_{j}}\right)=$ $\left(w_{0}, v_{0}\right)$ and $\left(w_{0}, v_{0}\right)$ is a solution of problem (1.1). Since $0<\left\|\left(x_{0}, y_{0}\right)\right\|<$ $R_{1}<\left\|\left(w_{0}, v_{0}\right)\right\|<R_{2}$ then $\left(x_{0}, y_{0}\right)$ and $\left(w_{0}, v_{0}\right)$ are two distinct positive solutions of problem (1.1).

Example 4.1. Consider the bvp:

$$
\begin{aligned}
& \left\{\begin{array}{l}
-D_{0^{+}}^{\frac{5}{4}}\left(D_{0^{+}}^{\frac{13}{4}} x(t)\right)^{\frac{1}{3}}=4 \sqrt{\pi}\left(\frac{8}{9} \delta_{1}\right)^{\frac{1}{3}} t^{2}(1-t)^{\frac{7}{4}} \frac{x^{2}+\ln (x+2)}{x}(x+y+1), \\
-D_{0^{+}}^{\frac{7}{4}}\left(D_{0^{+}}^{\frac{7}{2}} y(t)\right)^{\frac{1}{5}}=\left(1-2^{\frac{-1}{5}}\right) \frac{\sqrt{\pi}}{2}\left(\frac{6}{5} \delta_{2}\right)^{\frac{1}{5}} t^{4}(1-t)^{\frac{13}{4}} \frac{x^{3}+x+1}{x^{2}} e^{(x+y)^{\frac{1}{5}}}, \\
x(0)=x(1)=x^{\prime}(0)=x^{\prime}(1)=0, D_{0^{+}}^{\frac{13}{4}} x(0)=0, D_{0^{+}}^{\frac{13}{4}} x(1)=\frac{1}{2^{\frac{1}{4}}} D_{0^{+}}^{\frac{13}{4}} x\left(\frac{1}{2}\right), \\
y(0)=y(1)=y^{\prime}(0)=y^{\prime}(1)=0, D_{0^{+}}^{\frac{7}{2}} y(0)=0, D_{0^{+}}^{\frac{7}{4}} y(1)=2^{\frac{11}{4}} D_{0^{+}}^{\frac{7}{2}} y\left(\frac{1}{2}\right),
\end{array}\right. \\
& (4.5)
\end{aligned}
$$

where

$$
\begin{aligned}
f(t, x, y) & =(1-t)^{\frac{7}{4}} \frac{x^{2}+\ln (x+2)}{x}(x+y+1), g(t, x, y)=(1-t)^{\frac{13}{4}} \frac{x^{3}+x+1}{x^{2}} e^{(x+y)^{\frac{1}{5}}}, \\
a_{1}(t) & =4 \sqrt{\pi}\left(\frac{8}{9} \delta_{1}\right)^{\frac{1}{3}} t^{2}, a_{2}(t)=\left(1-2^{\frac{-1}{5}}\right) \frac{\sqrt{\pi}}{2}\left(\frac{6}{5} \delta_{2}\right)^{\frac{1}{5}} t^{4},\left(\delta_{1}, \delta_{2}>0\right),
\end{aligned}
$$

and $\phi_{1}(t)=t^{\frac{1}{3}}, \phi_{2}(t)=t^{\frac{1}{5}}$. Hence $\phi_{i}, i \in\{1,2\}$ are continuous homeomorphisms which are increasing and multiplicative with $\phi_{i}(0)=0$. Let us check Assumptions $\left(\mathcal{A}_{1}\right)-\left(\mathcal{A}_{4}\right)$ :

$$
\begin{aligned}
& \left(\mathcal{A}_{1}\right) \text { Let } m_{1}(t)=(1-t)^{\frac{7}{4}}, m_{2}(t)=(1-t)^{\frac{13}{4}}, q_{1}(x)=\frac{x^{2}+\ln (x+2)}{x} \\
& q_{2}(x)=\frac{x^{3}+x+1}{x^{2}}, \psi_{1}(x, y)=x+y+1, \psi_{2}(x, y)=e^{(x+y)^{\frac{1}{5}}}, p_{1}(x)=\frac{1}{x} \\
& p_{2}(x)=\frac{1}{x^{2}} . \text { Then } \\
& \quad \int_{0}^{1}(1-\tau)^{\alpha_{1}-1} a_{1}(\tau) m_{1}(\tau) p_{1}(c \gamma(\tau)) d \tau=\frac{4 \sqrt{\pi}\left(\frac{8}{9} \delta_{1}\right)^{\frac{1}{3}}}{c}<+\infty, \\
& \quad \int_{0}^{1}(1-\tau)^{\alpha_{2}-1} a_{2}(\tau) m_{2}(\tau) p_{2}\left(c \gamma(\tau) d \tau=\frac{\left(1-2^{\frac{-1}{5}}\right) \frac{\sqrt{\pi}}{2}\left(\frac{6}{5} \delta_{2}\right)^{\frac{1}{5}}}{c^{2}}<+\infty .\right.
\end{aligned}
$$


$\left(\mathcal{A}_{2}\right)$

$$
\begin{aligned}
& \sup _{c>0} \frac{c}{\sum_{i=1}^{2} \frac{M_{i}}{\Gamma\left(\beta_{i}\right)} \phi^{-1}\left(\lambda_{i} \frac{q_{i}(c)}{p_{i}(c)} \psi_{i}(c, c) \int_{0}^{1} a_{i}(\tau)(1-\tau)^{\alpha_{i}-1} m_{i}(\tau) p_{i}(\omega \gamma(\tau)) d \tau\right)} \\
\geq & \sup _{c>0} \frac{c}{\frac{\delta_{1}}{\omega^{3}}\left[8(2 c+1)\left(c^{2}+\ln (c+2)\right)\right]^{3}+\frac{\delta_{2}}{\omega^{10}}\left(c^{3}+c+1\right)^{5} e^{5(2 c)^{\frac{1}{5}}}} \\
\geq & \sup _{c>0} \frac{c}{\max \left\{\delta_{1}, \delta_{2}\right\}\left(\frac{1}{\omega^{3}}\left[8(2 c+1)\left(c^{2}+\ln (c+2)\right)\right]^{3}+\frac{1}{\omega^{10}}\left(c^{3}+c+1\right)^{5} e^{5(2 c)^{\frac{1}{5}}}\right)} .
\end{aligned}
$$

$\left(\mathcal{A}_{3}\right)$

$$
\begin{aligned}
& \min _{t \in[\sigma, 1-\sigma]} a_{1}(t)=\min _{t \in[\sigma, 1-\sigma]} 4 \sqrt{\pi}\left(\frac{8}{9} \delta_{1}\right)^{\frac{1}{3}} t^{2}=4 \sqrt{\pi}\left(\frac{8}{9} \delta_{1}\right)^{\frac{1}{3}} \sigma^{2} \neq 0, \\
& \min _{t \in[\sigma, 1-\sigma]} a_{2}(t)=\min _{t \in[\sigma, 1-\sigma]}\left(1-2^{\frac{-1}{5}}\right) \frac{\sqrt{\pi}}{2}\left(\frac{6}{5} \delta_{2}\right)^{\frac{1}{5}} t^{4}=\left(1-2^{\frac{-1}{5}}\right) \frac{\sqrt{\pi}}{2}\left(\frac{6}{5} \delta_{2}\right)^{\frac{1}{5}} \sigma^{4} \neq 0, \\
& \lim _{x+y \rightarrow+\infty} \inf _{t \in[\sigma, 1-\sigma]} \frac{f(t, x, y)}{\phi_{1}(x+y)}=\lim _{x+y \rightarrow+\infty} \inf _{t \in[\sigma, 1-\sigma]} \frac{(1-t)^{\frac{7}{4}} \frac{x^{2}+\ln (x+2)}{x}(x+y+1)}{(x+y)^{\frac{1}{3}}} \\
&=+\infty, \\
& \lim _{x+y \rightarrow+\infty} \inf _{t \in[\sigma, 1-\sigma]} \frac{g(t, x, y)}{\phi_{2}(x+y)}=\lim _{x+y \rightarrow+\infty} \inf _{t \in[\sigma, 1-\sigma]} \frac{(1-t)^{\frac{13}{4}} \frac{x^{3}+x+1}{x^{2}} e^{(x+y)^{\frac{1}{5}}}}{(x+y)^{\frac{1}{5}}} \\
&=+\infty .
\end{aligned}
$$

$\left(\mathcal{A}_{4}\right)$ For any $c>0$, we have

$$
\begin{array}{ll}
f(t, x, y) \geq \frac{\ln (2)}{c}(1-t)^{\frac{7}{4}}, & \forall t \in[0,1], \forall x, y \in(0, c], \\
g(t, x, y) \geq \frac{1}{c^{2}}(1-t)^{\frac{13}{4}}, & \forall t \in[0,1], \forall x, y \in(0, c] .
\end{array}
$$

If we choose $\delta_{1}, \delta_{2}$ such that

$$
0<\max \left\{\delta_{1}, \delta_{2}\right\}<\sup _{c>0} \frac{c}{\frac{1}{\omega^{3}}\left[8(2 c+1)\left(c^{2}+\ln (c+2)\right)\right]^{3}+\frac{1}{\omega^{10}}\left(c^{3}+c+1\right)^{5} e^{5(2 c)^{\frac{1}{5}}}},
$$

then all hypotheses of Theorem 4.1 are fulfilled. We conclude problem (4.5) has at least two positive solutions.

\section{Nonlinearities with two singularities}

In this section, we suppose that the nonlinearities $f, g:[0,1] \times \mathbf{R}_{*}^{+} \times \mathbf{R}_{*}^{+} \longrightarrow$ $\mathbf{R}^{+}$are continuous and satisfy

$\left(\mathcal{B}_{\mathbf{1}}\right)$ There exist $m_{i} \in C\left([0,1], \mathbf{R}^{+}\right), \psi_{i} \in C\left(\mathbf{R}^{+} \times \mathbf{R}^{+}, \mathbf{R}^{+}\right)$increasing functions for each argument and $k_{i}, p_{i}, q_{i}, l_{i} \in C\left(\mathbf{R}_{*}^{+}, \mathbf{R}^{+}\right)$such that $p_{i}, l_{i}$ 
are decreasing functions and $\frac{q_{i}}{p_{i} 0}, \frac{k_{i}}{l_{i}}$ are increasing functions $(i \in\{1,2\})$ with

$$
\begin{array}{ll}
f(t, x, y) \leq m_{1}(t) q_{1}(x) k_{1}(y) \psi_{1}(x, y), & \forall t \in[0,1] \times \mathbf{R}_{*}^{+} \times \mathbf{R}_{*}^{+} \\
g(t, x, y) \leq m_{2}(t) q_{2}(x) k_{2}(y) \psi_{2}(x, y), & \forall t \in[0,1] \times \mathbf{R}_{*}^{+} \times \mathbf{R}_{*}^{+}
\end{array}
$$

and for each $c, c^{\prime}>0$

$$
\int_{0}^{1}(1-\tau)^{\alpha_{2}-1} a_{i}(\tau) m_{i}(\tau) p_{i}(c \gamma(\tau)) l_{i}\left(c^{\prime} \gamma(\tau)\right) d \tau<+\infty .
$$

$\left(\mathcal{B}_{2}\right)$ For all $0<\omega<1$,

$$
\sup _{c>0} \frac{c}{\sum_{i=1}^{2} \frac{M_{i}}{\Gamma\left(\beta_{i}\right)} \phi_{i}^{-1}\left(\lambda_{i, c} \int_{0}^{1} a_{i}(\tau)(1-\tau)^{\alpha_{i}-1} m_{i}(\tau) p_{i}(\omega \gamma(\tau)) l_{i}(\omega \gamma(\tau)) d \tau\right)}>1,
$$

with

$$
\lambda_{i, c}=\lambda_{i} \frac{q_{i}(c)}{p_{i}(c)} \frac{k_{i}(c)}{l_{i}(c)} \psi_{i}(c, c) .
$$

$\left(\mathcal{B}_{3}\right)$ There exist $\sigma \in\left(0, \frac{1}{2}\right)$ and $F_{1}, F_{2} \in C\left(\left(\mathbf{R}_{*}^{+}\right)^{2}, \mathbf{R}^{+}\right)$, such that

$$
f(t, x, y) \geq F_{1}(x, y), g(t, x, y) \geq F_{2}(x, y), \quad \forall(t, x, y) \in[0,1] \times\left(\mathbf{R}_{*}^{+}\right)^{2},
$$

where

$$
\lim _{x+y \rightarrow+\infty} \inf _{t \in[\sigma, 1-\sigma]} \frac{F_{1}(x, y)}{\phi_{1}(x+y)} \geq l_{1}, \lim _{x+y \rightarrow+\infty} \inf _{t \in[\sigma, 1-\sigma]} \frac{F_{2}(x, y)}{\phi_{2}(x+y)} \geq l_{2},
$$

and

$$
l_{i}=1+\frac{\phi_{i}\left(\frac{\Gamma\left(\beta_{i}\right)}{2 c_{i}(1-2 \sigma)^{2}\left(\beta_{i}-2\right)}\right)}{\int_{\sigma}^{1-\sigma} a_{i}(\tau) H_{i}\left(d_{i}, \tau\right) d \tau}, i \in\{1,2\} \text { with } \min _{t \in[\sigma, 1-\sigma]} a_{i}(\tau) \neq 0
$$

and $c_{i}=\min _{(t, s) \in([\sigma, 1-\sigma])^{2}} k_{i}(t) L_{i}(s), H_{i}\left(d_{i}, \tau\right)=\min _{s \in[\sigma, 1-\sigma]} H_{i}(s, \tau)$.

$\left(\mathcal{B}_{\mathbf{4}}\right)$ For any $c>0$ and $\frac{1}{4}<\theta<\frac{1}{2}$, there exists $\rho_{c}, \rho_{c}^{*} \in C\left([0,1], \mathbf{R}^{+}\right)$such that $\rho_{c}(t)>0, \rho_{c}^{*}(t)>0$ on $\left(\theta-\frac{1}{4}, \theta\right)$ and

$$
\begin{aligned}
& f(t, x, y) \geq \rho_{c}(t), \quad \forall t \in[0,1], \forall x, y \in(0, c] \\
& g(t, x, y) \geq \rho_{c}^{*}(t), \quad \forall t \in[0,1], \forall x, y \in(0, c]
\end{aligned}
$$
by

Define two sequences of approximating functions $\left\{f_{n}\right\}_{n \geq 1}$ and $\left\{g_{n}\right\}_{n \geq 1}$

$$
\begin{array}{ll}
f_{n}(t, x, y)=f\left(t, \max \left\{\frac{1}{n}, x\right\}, \max \left\{\frac{1}{n}, y\right\}\right), & n \in\{1,2, \ldots\}, \\
g_{n}(t, x, y)=g\left(t, \max \left\{\frac{1}{n}, x\right\}, \max \left\{\frac{1}{n}, y\right\}\right), & n \in\{1,2, \ldots\} .
\end{array}
$$


For $(x, y) \in \mathcal{P}$, define the sequences of operators

$$
\begin{aligned}
& A_{n}(x, y)(t)=\int_{0}^{1} G_{1}(t, s) \phi_{1}^{-1}\left(\int_{0}^{1} H_{1}(s, \tau) a_{1}(\tau) f_{n}(\tau, x(\tau, y(\tau)) d \tau) d s\right. \\
& B_{n}(x, y)(t)=\int_{0}^{1} G_{2}(t, s) \phi_{2}^{-1}\left(\int_{0}^{1} H_{2}(s, \tau) a_{2}(\tau) g_{n}(\tau, x(\tau, y(\tau)) d \tau) d s\right.
\end{aligned}
$$

and

$$
T_{n}(x, y)=\left(A_{n}(x, y), B_{n}(x, y)\right)
$$

As for Proposition 3.1, we can prove

Proposition 5.1. Assume that condition $\left(\mathcal{B}_{1}\right)$ holds. Then for each $n \geq 1$, the operator $T_{n}: P \longrightarrow P$ is completely continuous.

Our main result in this section concerns the existence of two positive solutions.

Theorem 5.1. Assume that Assumptions $\left(\mathcal{B}_{1}\right)-\left(\mathcal{B}_{4}\right)$ hold. Then problem (1.1) has at least two positive solutions.

Proof. Step 1: An approximating solution. From condition $\left(\mathcal{B}_{2}\right)$, there exists $R_{1}>0$ such that, for all $0<\omega<1$

$$
\frac{R_{1}}{\sum_{i=1}^{2} \frac{M_{i}}{\Gamma\left(\beta_{i}\right)} \phi_{i}^{-1}\left(\lambda_{i, R_{1}} \int_{0}^{1} a_{i}(\tau)(1-\tau)^{\alpha_{i}-1} m_{i}(\tau) p_{i}(\omega \gamma(\tau)) l_{i}(\omega \gamma(\tau)) d \tau\right)}>1 .
$$

Let

$$
\Omega_{1}=\left\{(x, y) \in E:\|(x, y)\|<R_{1}\right\} .
$$

We claim that $(x, y) \neq \lambda T_{n}(x, y)$ for any $x \in \partial \Omega_{1} \cap \mathcal{P}, \lambda \in(0,1]$ and $n \geq n_{0} \geq \frac{1}{R_{1}}$. On the contrary, suppose that there exists $n_{1} \geq n_{0}$, $\left(x_{1}, y_{1}\right) \in \partial \Omega_{1} \cap \mathcal{P}$, and $\lambda_{1} \in(0,1]$ such that $\left(x_{1}, y_{1}\right)=\lambda_{1} T_{n_{1}}\left(x_{1}, y_{1}\right)$. 
As a consequence, we have the estimates:

$$
\begin{aligned}
\left\|x_{1}\right\|= & \left\|\lambda_{1} A_{n_{1}}\left(x_{1}, y_{1}\right)\right\| \\
\leq & \left\|A_{n_{1}}\left(x_{1}, y_{1}\right)\right\| \\
\leq & \left\|\int_{0}^{1} G_{1}(t, s) \phi_{1}^{-1}\left(\int_{0}^{1} a_{1}(\tau) H_{1}(s, \tau) f_{n_{1}}\left(\tau, x_{1}(\tau), y_{1}(\tau)\right) d \tau\right) d s\right\| \\
\leq & \frac{M_{1}}{\Gamma\left(\beta_{1}\right)} \phi_{1}^{-1}\left(\int _ { 0 } ^ { 1 } a _ { 1 } ( \tau ) \lambda _ { 1 } ( 1 - \tau ) ^ { \alpha _ { 1 } - 1 } f \left(\tau, \max \left\{\frac{1}{n_{1}}, x_{1}(\tau)\right\},\right.\right. \\
& \left.\max \left\{\frac{1}{n_{1}}, y_{1}(\tau)\right\}\right) d \tau \\
\leq & \frac{M_{1}}{\Gamma\left(\beta_{1}\right)} \phi_{1}^{-1}\left(\lambda_{1} \int_{0}^{1} a_{1}(\tau)(1-\tau)^{\alpha_{1}-1} m_{1}(\tau) q_{1}\left(\max \left\{\frac{1}{n_{1}}, x_{1}(\tau)\right\}\right)\right. \\
& \left.k_{1}\left(\max \left\{\frac{1}{n_{1}}, y_{1}(\tau)\right\}\right) \psi_{1}\left(\max \left\{\frac{1}{n_{1}}, x_{1}(\tau)\right\}, \max \left\{\frac{1}{n_{1}}, y_{1}(\tau)\right\}\right) d \tau\right) \\
\leq & \frac{M_{1}}{\Gamma\left(\beta_{1}\right)} \phi_{1}^{-1}\left(\lambda_{1} \int_{0}^{1} a_{1}(\tau)(1-\tau)^{\alpha_{1}-1} m_{1}(\tau) p_{1}\left(\max \left\{\frac{1}{n_{1}}, x_{1}(\tau)\right\}\right)\right. \\
& l_{1}\left(\max \left\{\frac{1}{n_{1}}, y_{1}(\tau)\right\}\right) \frac{q_{1}\left(\max \left\{\frac{1}{n_{1}}, x_{1}(\tau)\right\}\right)}{p_{1}\left(\max \left\{\frac{1}{n_{1}}, x_{1}(\tau)\right\}\right)} \frac{k_{1}\left(\max \left\{\frac{1}{n_{1}}, y_{1}(\tau)\right\}\right)}{\left.l_{1}\left(\max \frac{1}{n_{1}}, y_{1}(\tau)\right\}\right)} \\
& \left.\psi_{1}\left(\max \left\{\frac{1}{n_{1}}, x_{1}(\tau)\right\}, \max \left\{\frac{1}{n_{1}}, y_{1}(\tau)\right\}\right)\right) d \tau \\
\leq & \frac{M_{1}}{\Gamma\left(\beta_{1}\right)} \phi_{1}^{-1}\left(\lambda_{1} \frac{q_{1}\left(R_{1}\right)}{p_{1}\left(R_{1}\right)} \frac{k_{1}\left(R_{1}\right)}{l_{1}\left(R_{1}\right)} \psi_{1}\left(R_{1}, R_{1}\right)\right) \\
& \phi_{1}^{-1}\left(\int _ { 0 } ^ { 1 } a _ { 1 } ( \tau ) ( 1 - \tau ) ^ { \alpha _ { 1 } - 1 } m _ { 1 } ( \tau ) p _ { 1 } \left(\left(\frac{1}{n_{1}}\right) l_{1}\left(\left(\frac{1}{n_{1}}\right) d \tau\right)\right.\right. \\
\leq & \frac{M_{1}}{\Gamma\left(\beta_{1}\right)} \phi_{1}^{-1}\left(\lambda_{1} \frac{q_{1}\left(R_{1}\right)}{p_{1}\left(R_{1}\right)} \frac{k_{1}\left(R_{1}\right)}{l_{1}\left(R_{1}\right)} \psi_{1}\left(R_{1}, R_{1}\right)\right) \\
& \phi_{1}^{-1}\left(\int _ { 0 } ^ { 1 } a _ { 1 } ( \tau ) ( 1 - \tau ) ^ { \alpha _ { 1 } - 1 } m _ { 1 } ( \tau ) p _ { 1 } \left(\left(\frac{1}{n_{1}} \gamma(\tau)\right) l_{1}\left(\left(\frac{1}{n_{1}} \gamma(\tau)\right) d \tau\right) .\right.\right.
\end{aligned}
$$

Similarly,

$$
\begin{aligned}
\left\|y_{1}\right\| \leq & \left\|\lambda_{1} B_{n_{1}}\left(x_{1}, y_{1}\right)\right\| \\
\leq & \left\|B_{n_{1}}\left(x_{1}, y_{1}\right)\right\| \\
\leq & \left\|\int_{0}^{1} G_{2}(t, s) \phi_{2}^{-1}\left(\int_{0}^{1} a_{2}(\tau) H_{2}(s, \tau) g_{n_{1}}\left(\tau, x_{1}(\tau), y_{1}(\tau)\right) d \tau\right) d s\right\|, \\
\leq & \frac{M_{2}}{\Gamma\left(\beta_{2}\right)} \phi_{2}^{-1}\left(\int _ { 0 } ^ { 1 } a _ { 2 } ( \tau ) \lambda _ { 2 } ( 1 - \tau ) ^ { \alpha _ { 2 } - 1 } g \left(\tau, \max \left\{\frac{1}{n_{1}}, x_{1}(\tau)\right\},\right.\right. \\
& \left.\max \left\{\frac{1}{n_{1}}, y_{1}(\tau)\right\}\right) d \tau, \\
\leq & \frac{M_{2}}{\Gamma\left(\beta_{2}\right)} \phi_{2}^{-1}\left(\lambda_{2} \int_{0}^{1} a_{2}(\tau)(1-\tau)^{\alpha_{2}-1} m_{2}(\tau) q_{2}\left(\max \left\{\frac{1}{n_{1}}, x_{1}(\tau)\right\}\right)\right. \\
& \left.k_{2}\left(\max \left\{\frac{1}{n_{1}}, y_{1}(\tau)\right\}\right) \psi_{2}\left(\max \left\{\frac{1}{n_{1}}, x_{1}(\tau)\right\}, \max \left\{\frac{1}{n_{1}}, y_{1}(\tau)\right\}\right) d \tau\right) \\
\leq & \frac{M_{2}}{\Gamma\left(\beta_{2}\right)} \phi_{2}^{-1}\left(\lambda_{2} \frac{q_{2}\left(R_{1}\right)}{p_{2}\left(R_{1}\right)} \frac{k_{2}\left(R_{1}\right)}{l_{2}\left(R_{1}\right)} \psi_{2}\left(R_{1}, R_{1}\right)\right) \\
& \phi_{2}^{-1}\left(\int_{0}^{1} a_{2}(\tau)(1-\tau)^{\alpha_{2}-1} m_{2}(\tau) p_{2}\left(\frac{1}{n_{1}}\right) l_{2}\left(\frac{1}{n_{1}}\right) d \tau\right) \\
\leq & \frac{M_{2}}{\Gamma\left(\beta_{2}\right)} \phi_{2}^{-1}\left(\lambda_{2} \frac{q_{2}\left(R_{1}\right)}{p_{2}\left(R_{1}\right)} \frac{k_{2}\left(R_{1}\right)}{l_{2}\left(R_{1}\right)} \psi_{2}\left(R_{1}, R_{1}\right)\right) \\
& \phi_{2}^{-1}\left(\int_{0}^{1} a_{2}(\tau)(1-\tau)^{\alpha_{2}-1} m_{2}(\tau) p_{2}\left(\frac{1}{n_{1}} \gamma(\tau)\right) l_{2}\left(\frac{1}{n_{1}} \gamma(\tau)\right) d \tau\right) .
\end{aligned}
$$

Then

$$
\begin{aligned}
& \left\|\left(x_{1}, y_{1}\right)\right\| \\
\leq & \frac{M_{1}}{\Gamma\left(\beta_{1}\right)} \phi_{1}^{-1}\left(\lambda_{1, R_{1}} \int_{0}^{1} a_{1}(\tau) m_{1}(\tau)(1-\tau)^{\alpha_{1}-1} p_{1}\left(\frac{1}{n_{1}} \tau\right) l_{1}\left(\frac{1}{n_{1}} \gamma(\tau)\right) d \tau\right) \\
+ & \frac{M_{2}}{\Gamma\left(\beta_{2}\right)} \phi_{2}^{-1}\left(\lambda_{2, R_{1}} \int_{0}^{1} a_{2}(\tau) m_{2}(\tau)(1-\tau)^{\alpha_{2}-1} p_{2}\left(\frac{1}{n_{1}} \gamma(\tau)\right) l_{2}\left(\frac{1}{n_{1}} \gamma(\tau)\right) d \tau\right),
\end{aligned}
$$


which is a contradiction to (5.3). By Lemma 2.2, we deduce that

$$
i\left(T_{n}, \Omega_{1} \cap \mathcal{P}, \mathcal{P}\right)=1, \text { for all } n \in\left\{n_{0}, n_{0}+1, \ldots\right\} .
$$

Consequently there exists a sequence of fixed points $\left(x_{n}, y_{n}\right) \in \Omega_{1} \cap \mathcal{P}$ such that $T_{n}\left(x_{n}, y_{n}\right)=\left(x_{n}, y_{n}\right), \forall n \geq n_{0}$ and $\left\|\left(x_{n}, y_{n}\right)\right\|<R_{1}$.

\section{Step 2: a compactness argument.}

(a) Since $\left\|\left(x_{n}, y_{n}\right)\right\|<R_{1}$, by $\left(\mathcal{B}_{4}\right)$ there exist functions $\rho_{R_{1}}, \rho_{R_{1}}^{*} \in C\left([0,1], \mathbf{R}^{+}\right)$ such that

$$
\begin{array}{ll}
f_{n}(t, x(t), y(t)) \geq \rho_{R_{1}}(t), & \forall t \in[0,1], \\
g_{n}(t, x(t), y(t)) \geq \rho_{R_{1}}^{*}(t), & \forall t \in[0,1] .
\end{array}
$$

Then

$$
\begin{aligned}
& x_{n}(t)=A_{n}\left(x_{n}, y_{n}\right)(t) \\
& \geq \int_{0}^{1} G_{1}(t, s) \phi_{1}^{-1}\left(\int_{0}^{1} a_{1}(\tau) H_{1}(s, \tau) f_{n}\left(\tau, x_{n}(\tau), y_{n}(\tau)\right) d \tau\right) d s \\
& \geq \int_{\theta}^{1-\theta} \frac{\left(\beta_{1}-2\right) K_{1}(t) L_{1}(s)}{\Gamma\left(\beta_{1}\right)} \phi_{1}^{-1}\left(\int_{\theta-\frac{1}{4}}^{\theta} a_{1}(\tau) H_{1}(s, \tau) \rho_{R_{1}}(\tau) d \tau\right) d s \\
& \geq \frac{\phi_{1}^{-1}\left(\frac{1}{\Gamma\left(\alpha_{1}\right)}\right)\left(\beta_{1}-2\right) K_{1}(t)}{\Gamma\left(\beta_{1}\right)} \\
& \int_{\theta}^{1-\theta} L_{1}(s) \phi_{1}^{-1}\left(\int_{\theta-\frac{1}{4}}^{\theta} a_{1}(\tau)\left[[s(1-\tau)]^{\alpha_{1}-1}-(s-\tau)^{\alpha_{1}-1}\right] \rho_{R_{1}}(\tau) d \tau\right) d s \\
& y_{n}(t)=B_{n}\left(x_{n}, y_{n}\right)(t) \\
& \geq \int_{0}^{1} G_{2}(t, s) \phi_{2}^{-1}\left(\int_{0}^{1} a_{2}(\tau) H_{2}(s, \tau) g_{n}\left(\tau, x_{n}(\tau), y_{n}(\tau)\right) d \tau\right) d s \\
& \geq \int_{\theta}^{1-\theta} \frac{\left(\beta_{2}-2\right) K_{2}(t) L_{2}(s)}{\Gamma\left(\beta_{2}\right)} \phi_{2}^{-1}\left(\int_{\theta-\frac{1}{4}}^{\theta} a_{2}(\tau) H_{2}(s, \tau) g_{n}\left(\tau, x_{n}(\tau), y_{n}(\tau)\right) d \tau\right) d s \\
& \geq \frac{\phi_{2}^{-1}\left(\frac{1}{\Gamma\left(\alpha_{2}\right)}\right)\left(\beta_{2}-2\right) K_{2}(t)}{\Gamma\left(\beta_{2}\right)} \\
& \int_{\theta}^{1-\theta} L_{2}(s) \phi_{2}^{-1}\left(\int_{\theta-\frac{1}{4}}^{\theta} a_{2}(\tau)\left[[s(1-\tau)]^{\alpha_{2}-1}-(s-\tau)^{\alpha_{2}-1}\right] \rho_{R_{1}}^{*}(\tau) d \tau\right) d s .
\end{aligned}
$$

Let the positive numbers

$$
\begin{gathered}
c_{1}^{*}=\frac{\phi_{1}^{-1}\left(\frac{1}{\Gamma\left(\alpha_{1}\right)}\right)\left(\beta_{1}-2\right)}{\Gamma\left(\beta_{1}\right)} \\
\int_{\theta}^{1-\theta} L_{1}(s) \phi_{1}^{-1}\left(\int_{\theta-\frac{1}{4}}^{\theta} a_{1}(\tau)\left[[s(1-\tau)]^{\alpha_{1}-1}-(s-\tau)^{\alpha_{1}-1}\right] \rho_{R_{1}}(\tau) d \tau\right) d s
\end{gathered}
$$

and

$$
c_{2}^{*}=\frac{\phi_{2}^{-1}\left(\frac{1}{\Gamma\left(\alpha_{2}\right)}\right)\left(\beta_{2}-2\right)}{\Gamma\left(\beta_{2}\right)}
$$




$$
\int_{\theta}^{1-\theta} L_{2}(s) \phi_{2}^{-1}\left(\int_{\theta-\frac{1}{4}}^{\theta} a_{2}(\tau)\left[[s(1-\tau)]^{\alpha_{2}-1}-(s-\tau)^{\alpha_{2}-1}\right] \rho_{R_{1}}^{*}(\tau) d \tau\right) d s .
$$

Then, $x_{n}(t) \geq c_{1}^{*} \gamma(t), y_{n}(t) \geq c_{2}^{*} \gamma(t), \quad \forall t \in[0,1], \forall n \geq n_{0}$.

(b) For any $t, t^{\prime} \in[0,1]\left(t>t^{\prime}\right)$, we have the following estimates:

$$
\begin{aligned}
& \left|x_{n}(t)-x_{n}\left(t^{\prime}\right)\right| \\
\leq & \int_{0}^{1}\left|G_{1}(t, s)-G_{1}\left(t^{\prime}, s\right)\right| \phi_{1}^{-1}\left(\int_{0}^{1} a_{1}(\tau) H_{1}(s, \tau) f_{n}\left(\tau, x_{n}(\tau), y_{n}(\tau)\right) d \tau\right) d s \\
\leq & \int_{0}^{1}\left|G_{1}(t, s)-G_{1}\left(t^{\prime}, s\right)\right| \phi_{1}^{-1}\left(\lambda_{1, R_{1}} \int_{0}^{1} a_{1}(\tau) m_{1}(\tau)(1-\tau)^{\alpha_{1}-1} p_{1}\right. \\
& \left(c_{1}^{*} \gamma(\tau)\right) l_{1}\left(c_{2}^{*} \gamma(\tau)\right) d \tau .
\end{aligned}
$$

Similarly,

$$
\begin{aligned}
& \left|y_{n}(t)-y_{n}\left(t^{\prime}\right)\right| \\
\leq & \int_{0}^{1}\left|G_{2}(t, s)-G_{2}\left(t^{\prime}, s\right)\right| \phi_{2}^{-1}\left(\int_{0}^{1} a_{2}(\tau) H_{2}(s, \tau) g_{n}\left(\tau, x_{n}(\tau), y_{n}(\tau)\right) d \tau\right) d s \\
\leq & \int_{0}^{1}\left|G_{2}(t, s)-G_{2}\left(t^{\prime}, s\right)\right| \phi_{2}^{-1}\left(\lambda_{2, R_{1}} \int_{0}^{1} a_{2}(\tau) m_{2}(\tau)(1-\tau)^{\alpha_{1}-1} p_{2}\right. \\
& \left(c_{1}^{*} \gamma(\tau)\right) l_{2}\left(c_{2}^{*} \gamma(\tau)\right) d \tau .
\end{aligned}
$$

We conclude that $\left\{\left(x_{n}, y_{n}\right)\right\}_{n \geq n_{0}}$ has a convergent subsequence $\left\{\left(x_{n_{k^{\prime}}}, y_{n_{k^{\prime}}}\right)\right\}_{k^{\prime} \geq 1}$ such that $\lim _{k^{\prime} \rightarrow+\infty}\left(x_{n_{k^{\prime}}}, y_{n_{k^{\prime}}}\right)=\left(x^{*}, y^{*}\right)$ such that $x^{*} \geq c_{1}^{*} \gamma(t)$ and $y^{*} \geq$ $c_{2}^{*} \gamma(t)$. Consequently $\left(x^{*}, y^{*}\right)$ is a positive solution of problem (1.1). Arguing ass in the proof of Theorem 4.1, we can show by condition $\left(\mathcal{B}_{3}\right)$ that problem (1.1) admits a second positive solution.

Example 5.1. Consider the bvp

$$
\left\{\begin{array}{l}
-D_{0^{+}}^{\frac{5}{4}}\left(\phi_{1}\left(D_{0^{+}}^{\frac{13}{4}} x(t)\right)\right)=\frac{9 \sqrt{\pi}}{32} t^{4}\left(t^{2}+1\right)(1-t)^{\frac{15}{4} \frac{\left(x^{2}+1\right)\left(y^{2}+1\right)}{x y}\left((x+y)^{3}+1\right),} \\
-D_{0^{+}}^{\frac{7}{4}}\left(\phi_{2}\left(D_{0^{+}}^{\frac{7}{2}} y(t)\right)\right)=\sqrt{\pi} t^{8}(t+1)(1-t)^{\frac{29}{4}} \frac{\left(x^{3}+1\right)\left(y^{3}+1\right)\left((x+y) e^{(|x|+|y|)}+1\right)}{x^{2} y^{2}}, \\
x(0)=x(1)=x^{\prime}(0)=x^{\prime}(1)=0, D_{0^{+}}^{\frac{13}{4}} x(0)=0, D_{0^{+}}^{\frac{13}{4}} x(1)=\frac{2^{\frac{-7}{8}}}{\sqrt{\delta}} D_{0^{+}}^{\frac{13}{4}} x\left(\frac{1}{2}\right), \\
y(0)=y(1)=y^{\prime}(0)=y^{\prime}(1)=0, D_{0^{+}}^{\frac{7}{2}} y(0)=0, D_{0^{+}}^{\frac{7}{4}} y(1)=\frac{2^{-\frac{5}{8}}}{\sqrt{\delta}} D_{0^{+}}^{\frac{7}{2}} y\left(\frac{1}{2}\right),
\end{array}\right.
$$

where

$$
f(t, x, y)=\frac{\left(t^{2}+1\right)\left(x^{2}+1\right)\left(y^{2}+1\right)}{x y}\left((x+y)^{3}+1\right),
$$




$$
\begin{aligned}
g(t, x, y) & =\frac{(t+1)\left(x^{3}+1\right)\left(y^{3}+1\right)\left((x+y) e^{(|x|+|y|)}+1\right)}{x^{2} y^{2}}, \\
a_{1}(t) & =\frac{9 \sqrt{\pi}}{32} t^{4}(1-t)^{\frac{15}{4}}, a_{2}(t)=\sqrt{\pi} t^{8}(1-t)^{\frac{29}{4}}
\end{aligned}
$$

and $\phi_{1}(t)=\phi_{2}(t)=\delta t|t|, \delta>0$. Hence for $i \in\{1,2\}$, the functions $\phi_{i}$ are continuous homeomorphisms which are increasing, multiplicative with $\phi_{i}(0)=0$ and $\phi_{i}^{-1}(t)=\frac{1}{\sqrt{\delta}} t|t|^{\frac{-1}{2}}$. Next, we check Assumptions $\left(\mathcal{B}_{1}\right)$ and $\left(\mathcal{B}_{4}\right)$ :

$\left(\mathcal{B}_{1}\right)$ Let

$$
\begin{gathered}
m_{1}(t)=t^{2}+1, m_{2}(t)=t+1, q_{1}(x)=\frac{x^{2}+1}{x}, q_{2}(x)=\frac{x^{3}+1}{x^{2}} \\
k_{1}(y)=\frac{y^{2}+1}{y}, k_{2}(y)=\frac{y^{3}+1}{y^{2}}, \psi_{1}(x, y)=(x+y)^{3}+1, \\
\psi_{2}(x, y)=(x+y) e^{(|x|+|y|)}+1, p_{1}(x)=\frac{1}{x}, p_{2}(x)=\frac{1}{x^{2}}, \\
l_{1}(y)=\frac{1}{y}, l_{2}(y)=\frac{1}{y^{2}} .
\end{gathered}
$$

Then we have

$$
\begin{aligned}
& \int_{0}^{1}(1-\tau)^{\alpha_{1}-1} a_{1}(\tau) m_{1}(\tau) p_{1}(c \gamma(\tau)) l_{1}\left(c^{\prime} \gamma(\tau)\right) d \tau=\frac{3 \sqrt{\pi}}{8 c c^{\prime}}<+\infty, \\
& \int_{0}^{1}(1-\tau)^{\alpha_{2}-1} a_{2}(\tau) m_{2}(\tau) p_{2}(c \gamma(\tau)) l_{2}\left(c^{\prime} \gamma(\tau)\right) d \tau=\frac{3 \sqrt{\pi}}{2 c^{2} c^{\prime 2}}<+\infty .
\end{aligned}
$$

$\left(\mathcal{B}_{2}\right)$

$$
\begin{aligned}
& \sup _{c>0} \frac{c}{\sum_{i=1}^{2} \frac{M_{i}}{\Gamma\left(\beta_{i}\right)} \phi_{i}^{-1}\left(\lambda_{i, c} \int_{0}^{1} a_{i}(\tau) m_{i}(\tau)(1-\tau)^{\alpha_{i}-1} p_{i}(\omega \gamma(\tau)) l_{i}(\omega \gamma(\tau)) d \tau\right)} \\
\geq & \sup _{c>0} \frac{\sqrt{\delta} \min \left\{\omega, \omega^{2}\right\} c}{2 \sqrt{(2 c)^{3}+1}\left(c^{2}+1\right)+2 \sqrt{\left(c^{3}+1\right)^{2}\left(2 c e^{2 c}+1\right)}} .
\end{aligned}
$$

$\left(\mathcal{B}_{3}\right)$

$$
\begin{aligned}
\min _{t \in[\sigma, 1-\sigma]} a_{1}(t) & =\min _{t \in[\sigma, 1-\sigma]} \frac{9 \sqrt{\pi}}{32} t^{4}(1-t)^{\frac{15}{4}} \\
& \geq \frac{9 \sqrt{\pi}}{32} \sigma^{\frac{31}{4}} \neq 0, \\
\min _{t \in[\sigma, 1-\sigma]} a_{2}(t)=\min _{t \in[\sigma, 1-\sigma]} \sqrt{\pi} t^{8}(1-t)^{\frac{29}{4}} \geq \sqrt{\pi} \sigma^{\frac{65}{4}} \neq 0, & \\
\lim _{x+y \rightarrow+\infty} \inf _{t \in[\sigma, 1-\sigma]} \frac{F_{1}(x, y)}{\phi_{1}(x+y)} & =\lim _{x+y \rightarrow+\infty} \inf _{t \in[\sigma, 1-\sigma]} \frac{\frac{\left(x^{2}+1\right)\left(y^{2}+1\right)\left((x+y)^{3}+1\right)}{x y}}{\delta(x+y)|x+y|} \\
& =+\infty
\end{aligned}
$$




$$
\begin{aligned}
\lim _{x+y \rightarrow+\infty} \inf _{t \in[\sigma, 1-\sigma]} \frac{F_{2}(x, y)}{\phi_{2}(x+y)} & =\lim _{x+y \rightarrow+\infty} \inf _{t \in[\sigma, 1-\sigma]} \frac{\left(x^{3}+1\right)\left(y^{3}+1\right)\left((x+y) e^{(|x|+|y|)}+1\right)}{\delta(x y)^{2}(x+y)|x+y|} \\
& =+\infty
\end{aligned}
$$

$\left(\mathcal{B}_{4}\right)$

$$
\begin{array}{cl}
f(t, x, y) \geq \frac{t^{2}+1}{c^{2}}, & \forall t \in[0,1], \forall x, y \in(0, c] \\
g(t, x, y) \geq \frac{t+1}{c^{4}}, \quad \forall t \in[0,1], \forall x, y \in(0, c] &
\end{array}
$$

Let us pick some $\delta$ such that

$$
\delta>\left(\sup _{c>0} \frac{\min \left\{\omega, \omega^{2}\right\} c}{2 \sqrt{(2 c)^{3}+1}\left(c^{2}+1\right)+2\left(c^{3}+1\right) \sqrt{\left.2 c e^{2 c}+1\right)}}\right)^{-2} .
$$

Then the hypotheses of Theorem 5.1 are satisfied and problem (5.5) has at least two positive solutions.

\section{Acknowledgments.}

Research supported by the Direction Générale de la Recherche Scientifique et de Développement Technologique in Algeria. We would like to thank the anonymous referee for his/her careful read- ing and helpful suggestions which led to an improvement of the original manuscript.

\section{References}

[1] R. P. Agarwal, M. Meehan, and D. O'Regan, Fixed point theory and applications. Cambridge: Cambridge University Press, 2009, doi: 10.1017/ CBO9780511543005

[2] C. Cordeanu, Integral equations and stability of feedback systems. New York, NY: Academic Press, 1973.

[3] K. Deimling, Nonlinear Functional Analysis. Berlin: Springer, 1985, doi: 10.1007/978-3-662-00547-7

[4] S. Djebali and O. Saifi, "Positive solutions for singular $\phi$-Laplacian BVPs on the positive half-line", Electronic journal of qualitative theory of differential equations, vol. 2009, Art ID. 56, 2009, doi: 10.14232/ ejqtde.2009.1.56 
[5] S. Djebali and O. Saifi, "Multiple positive solutions for singular $\phi$ -Laplacian BVPs with derivative depending nonlinearity on $\mathbf{R}^{+"}$, Dynamic systems applications, vol. 21, no. 1, pp. 93-119, 2012.

[6] X. Hao, H. Wang, L. Liu, and Y. Cui, "Positive solutions for a system of nonlinear fractional nonlocal boundary value problems with parameters and p-Laplacian operator", Boundary value problems, vol. 2017, no. 1, pp. Art ID. 182, 2017, doi: 10.1186/ s13661-017-0915-5

[7] A. A. Kilbas, H. M. Srivastava, and J. J. Trujillo, Theory and applications of fractional differential equations. Amsterdam: Elsevier, 2006.

[8] R. L Magin, Fractional calculus in bioengineering. Redding, CT: Begell, 2006.

[9] I. Podlubny, Fractional differential equations: an introduction to fractional derivatives, fractional differential equations, to methods of their solution and some of their applications. San Diego, CA: Academic Press, 1999.

[10] J. Sabatier, O. P. Agrawal, and J. A. Tenreiro Machado, Advances in fractional calculus: theoretical developments and applications in physics and engineering. Dordrecht: Springer, 2007.

[11] S. G. Samko, O. I. Marichev, and A. A. Kilbas, Fractional integrals and derivatives: theory and applications. Yverdon: Gordon and Breach, 1993. [On line]. Available: https:/ / bit.ly/ 3qbTvdq

[12] J. F. Xu, and W. Dong, "Existence and uniqueness of positive solutions of a fractional boundary value problem with p-Laplacian operator", Acta mathematica. Sinica (Chinese Series), vol. 59, no. 3, pp. 385-396, 2016. [On line]. Available: https:/ / bit.ly/ 2Ptalbf

[13] F. Yan, M. Zuo, and X. Hao, "Positive solution for a fractional singular boundary value problem with p-Laplacian operator," Boundary value problems, vol. 2018, Art. ID. 51, 2018, doi: 10.1186/ s13661-018-0972-4

[14] G. M. Zaslavsky, Hamiltonian chaos and fractional dynamics. Oxford: Oxford University Press, 2008. 